
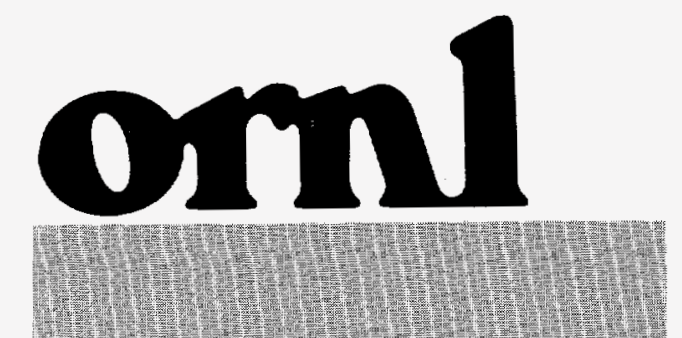

OAK RIDGE

NATIONAL

LABORATORY

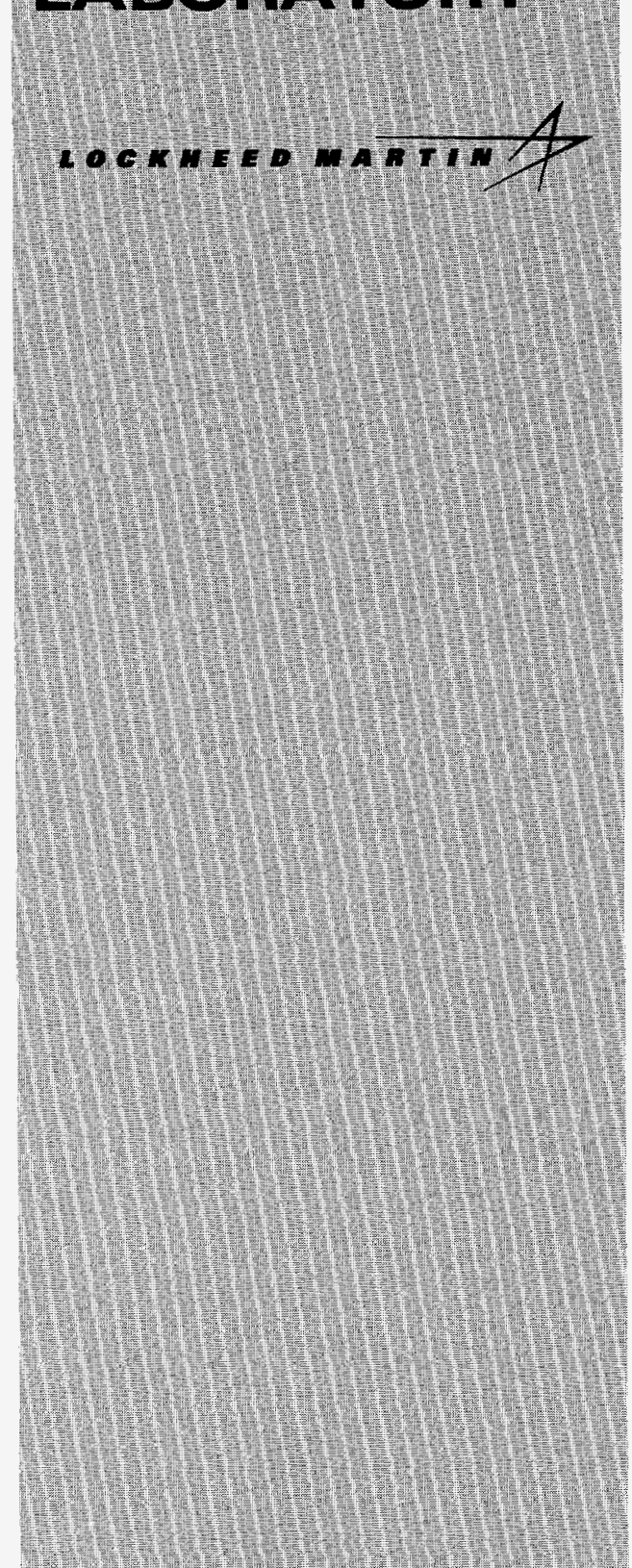

MANGEO AMO OPERATCO BY

LOCKHEED MARTY ENERGY RESEARCH CORPORATION FOA THE UTTEO STATES

DEPARTIENT OF ENERGY

\section{RECEIVED}

MAR 161998

OSTI

ORNL/TM-13590

\author{
Martin A. Hunt \\ Leon N. Klatt \\ David H. Thompson \\ Dianna J. Trent \\ James R. Younkin \\ Oak Ridge National Laboratory \\ and \\ Don E. Dale \\ Kelly Hakonson \\ Charles Rzeszutko \\ Los Alamos National Laboratory
}


This report has been reproduced directly from the best available copy.

Available to DOE and DOE contractors from the Office of Scientific and Technical Information, P.O. Box 62, Oak Ridge, TN 37831; prices available from (423) 576-8401, FTS 626-8401.

This report was prepared as an account of work sponsored by an agency of the United States Government. Neither the United States Government nor any agency thereof, nor any of their employees, makes any warranty, express or implied, or assumes any legal liability or responsibility for the accuracy, completeness, or usefuiness of any information, apparatus, product, or process disclosed, or represents that its use would not infringe privately owned rights. Reference herein to any specific commercial product, process, or service by trade name, trademark, manufacturer, or otherwise, does not necessarily constitute or imply its endorsement, recommendation, or favoring by the United States Government or any agency thereof. The views and opinions of authors expressed herein do not necessarily state or reflect those of the United States Government or any agency thereof. 


\section{DISCLAIMER}

Portions of this document may be illegible electronic image products. Images are produced from the best available original document. 


\title{
DEVELOPMENT TESTING OF THE CHEMICAL ANALYSIS AUTOMATION POLYCHLORINATED BIPHENYL STANDARD ANALYSIS METHOD DURING SURFACE SOIL SAMPLING AT THE DAVID WITHERSPOON 1630 SITE
}

\author{
Oak Ridge National Laboratory \\ P.O. Box 2008 \\ Oak Ridge, TN 37831-6285 \\ Don E. Dale, Kelly Hakonson, and Charles Rzeszutko \\ Los Alamos National Laboratory \\ P.O. Box 1663 \\ Los Alamos, NM 87545
}

Martin A. Hunt, Leon N. Klatt, David H. Thompson, Dianne J. Trent, and James R. Younkin

Date Published: February 1998

Prepared for the

U.S. Department of Energy, Office of Science and Technology

Robotics Technology Development Program - Chemical Analysis Automation

\author{
Prepared by \\ OAK RIDGE NATIONAL LABORATORY \\ Oak Ridge, Tennessee 37831-6285 \\ Managed by \\ LOCKHEED MARTIN ENERGY RESEARCH CORP. \\ for the \\ U.S. DEPARTMENT OF ENERGY \\ under contract DE-AC05-960R22464
}




\section{CONTENTS}

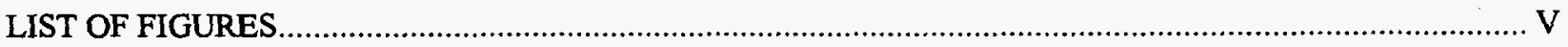

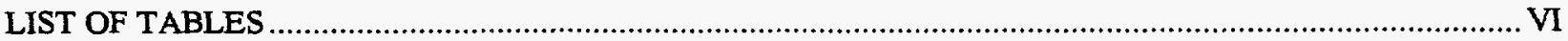

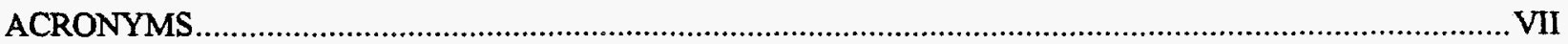

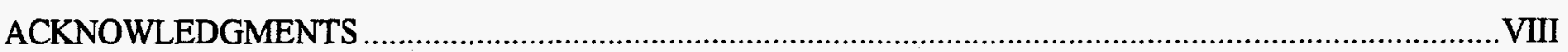

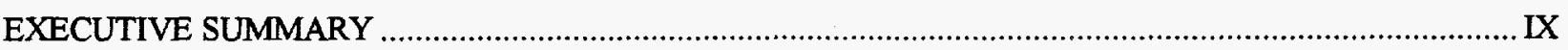

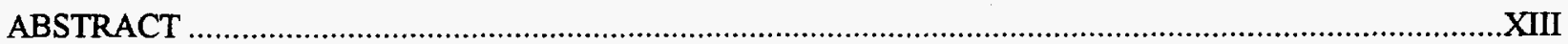

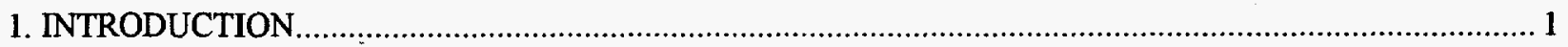

2. DESCRIPTION OF THE CHEMICAL ANALYSIS AUTOMATION PARADIGM ….............................. 2

3. DESCRIPTION OF THE POLYCHLORINATED BIPHENYL STANDARD ANALYSIS METHOD............... 6

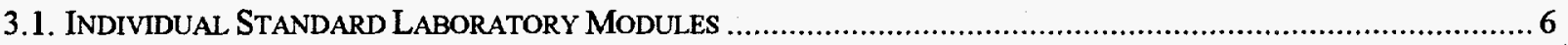

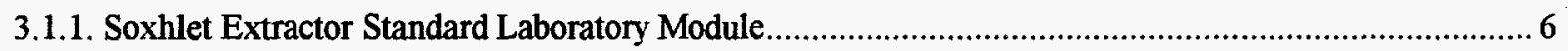

3.1.2. High-Volume Concentrator Standard Laboratory Module ............................................................. 6

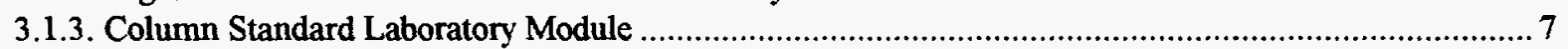

3.1.4. Gas Chromatograph Standard Laboratory Module ..................................................................... 7

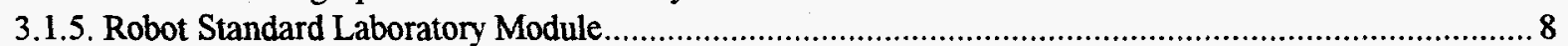

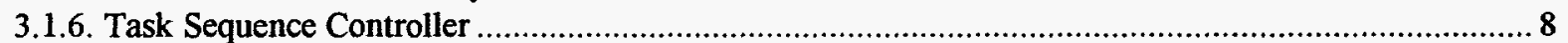

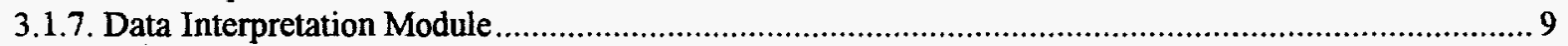

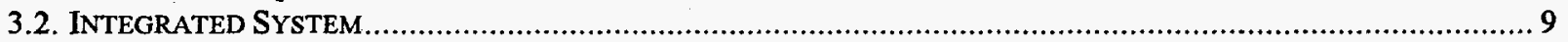

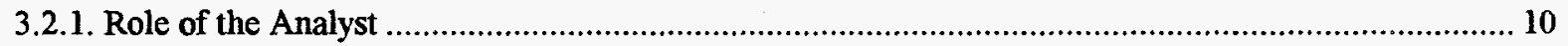

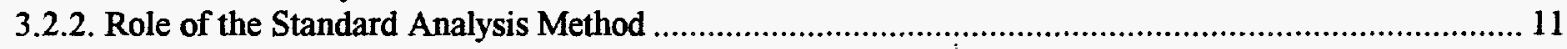

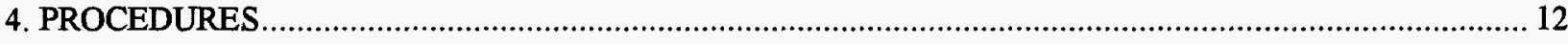

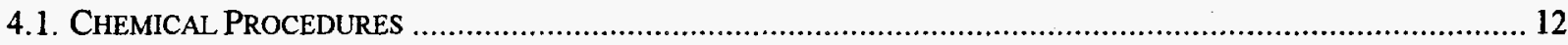

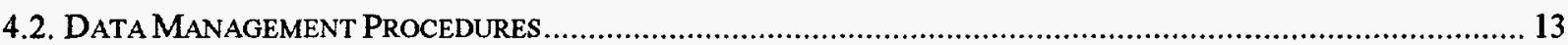

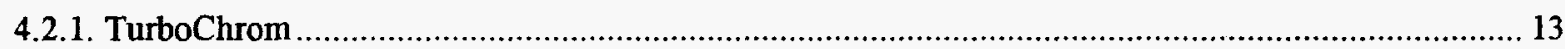

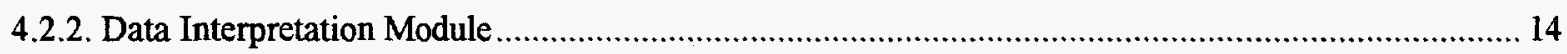

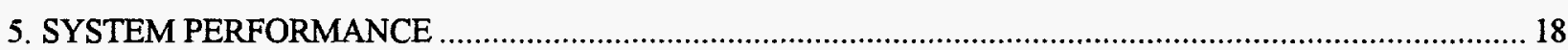

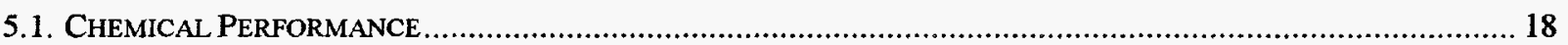

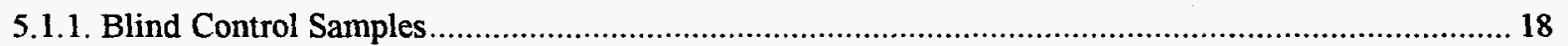

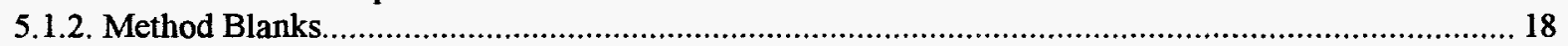

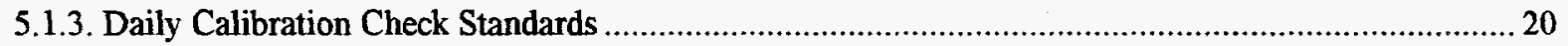

5.1.4. Matrix Spike and Matrix Spike Duplicate Samples ....................................................................... 21

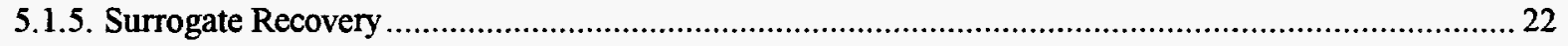

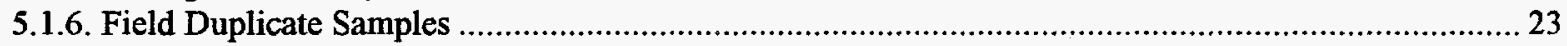

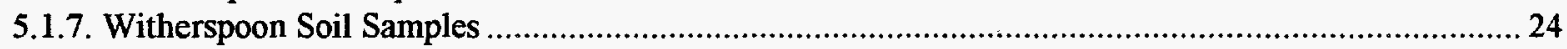

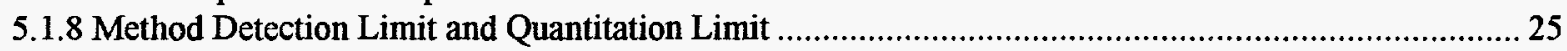

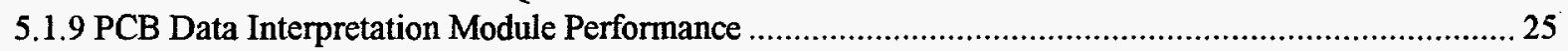

5.2. SYSTEM PERFORMANCE-HARDWARE SOFTWARE ERRORS AND ALARMS .................................................. 31

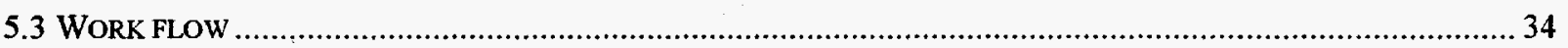

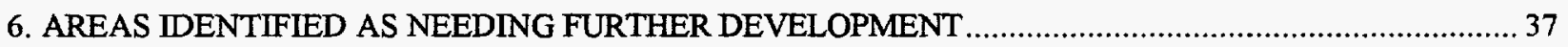


7. CONCLUSIONS.

APPENDIX A. SUMMARY OF THE TYPES OF ALARMS AND ERRORS ENCOUNTERED DURING THE WITHERSPOON PROJECT.

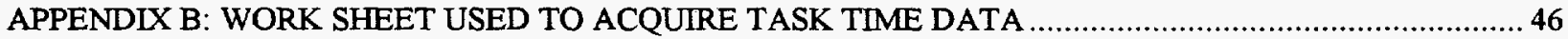

APPENDIX C: WORK SHEET USED TO ACQUIRE WORK-FLOW DATA ….........................................48 


\section{LIST OF FIGURES}

Figure

2.1 Block diagram representation of CAA paradigm

3.1 Graphical representation of the PCB SAM

3.2 PCB SAM configuration for the Witherspoon test .11

4.1 Block diagram of DIM fuzzy logic operations 16

5.1 System performance on daily calibration standards .21

5.2 Histogram distribution of PCBs in Witherspoon soil samples .24

5.3 Histogram of CAA PCB concentration difference .25

5.4 DIM performance on daily calibration standards .27

5.5 System errors and alarms by category .32

5.6 Summary of SLM errors and alarms 33

5.7 Graphical representation of work flow for Analyst 1 35

5.8 Graphical representation of work flow for Analyst 2 36

5.9 Preliminary cost projections for CAA PCB system .36 


\section{LIST OF TABLES}

Table

4.1 Fuzzy logic summary table

5.1 System performance on blind QC samples

5.2 System performance on method blanks 19

5.3 System performance on daily calibration standards. .20

5.4 System performance on matrix spikes and spike duplicates. .22

5.5 System performance on surrogate recovery 23

5.6 System performance on field duplicates 23

5.7 Summary of paired $t$-test comparing CAA with the commercial laboratory 24

5.8 Aroclor 1242 in blind QC samples .27

5.9 Aroclor 1254 in blind QC samples 28

5.10 Aroclor 1260 in blind QC samples. 28

5.11 Surrogate recovery in soil samples as determined by the DIM and TC4 software systems .29

5.12 Comparison of Aroclor identification in soil samples as determined by the DIM and $\mathrm{TC} 4$ software system. .30

5.13 Individual Aroclor content in soil samples as determined by the DIM and TC4 software systems. 


\section{ACRONYMS}

AIA

AIM

ANN

CAA

CI

DCBP

DIM

DOE

DWI

ECD

EPA

EQL

GC

GC-MS

$\mathrm{HCI}$

HVC

IEEE

ILUO

LANL

LIMS

LUO

MDL

MLRP

PCB

PCRP

PCRR

QA

QC

RPD

SAM

SLE

SLM

SSM

TSC
Analytical Instrument Association

Analytical Instrument Module

artificial neural network

Chemical Analysis Automation

confidence interval

decachlorbiphenyl

data interpretation module

U.S. Department of Energy

David Witherspoon, Inc.

electron capture detector

U.S. Environmental Protection Agency

estimated quantitation limit

gas chromatograph

gas chromatography mass spectrometer

human computer interface

high-volume concentrator

Institute of Electronic Electrical Engineering

intra-laboratory unit operation

Los Alamos National Laboratory

Laboratory Information Management System

laboratory unit operation

method detection limit

multiple linear regression-peak area data

polychlorinated biphenyl

principal component regression-peak area data

principal component regression-raw chromatogram data

quality assurance

quality control

relative percent difference

standard analysis method

simultaneous linear equations

standard laboratory module

service support module

task sequence controller 


\section{ACKNOWLEDGMENTS}

This research was sponsored by the Office of Science and Technology, U.S. Department of Energy, under contract DE-AC05-960R22464 with Lockheed Martin Energy Research Corporation.

We thank Dr. Linton Yarbroubh, II, Robotics Technology Development Program, for his continued support of the Chemical Analysis Automation project and for providing the funding necessary to complete the testing associated with the David Witherspoon 1630 Site.

The Chemical Analysis Automation project team thanks Andrea Perkins and David Cardin of the Oak Ridge Operations Office, U.S. Department of Energy, for providing the opportunity to work with the David Witherspoon 1630 Site project. The opportunity to process a large set of environmental samples provided the project team with an invaluable testing opportunity.

The cooperation of Thomas Goode and Michael Houk, Jacobs Engineering, Oak Ridge, Tennesse, is gratefully acknowledged.

The assistance of Carol Scott and Jeff Barnett in the areas of waste management and facility support is gratefully acknowledged. 


\section{EXECUTIVE SUMMARY}

The Chemical Analysis Automation (CAA) project is developing standardized, software-driven, site-deployable robotic laboratory systems with the objective of lowering the per-sample analysis cost, decreasing sample turnaround time, and minimizing human exposure to hazardous and radioactive materials associated with DOE remediation projects. The first integrated system developed by the CAA project is designed to determine polychlorinated biphenyls (PCB) content in soil matrices. A demonstration and development testing of this system was conducted in conjuction with surface soil characterization activities at the David Witherspoon 1630 Site in Knoxville, Tennessee.

The PCB system consists of five hardware standard laboratory modules (SLMs), one software SLM, the task sequence controller (TSC), and the human-computer interface (HCI). Four of the hardware SLMs included a four-channel Soxhlet extractor, a high-volume concentrator, a column cleanup, and a gas chromatograph. These SLMs performed the sample preparation and measurement steps within the total analysis protocol. The fifth hardware module was a robot that transports samples between the SLMs and the required consumable supplies to the SLMs. The software SLM is an automated data interpretation module that receives raw data from the gas chromatograph SLM and analyzes the data to yield the analyte information. The TSC is a software system that provides the scheduling, management of system resources, and the coordination of all SLM activities. The HCI is a graphical user interface that presents the automated laboratory to the analyst in terms of the analytical procedures and methods. Human control of the automated laboratory is accomplished via the HCI. Sample information required for processing by the automated laboratory is entered through the HCI. Information related to the sample and the system status is presented to the analyst via graphical icons.

The demonstration and development testing of the automated PCB laboratory was conducted according to and evaluated against the benchmark methods defined in the U.S. Environmental Protection Agency "Test Methods for Evaluating Solid Waste, Physical/Chemical Methods, SW-846" manual. The specific methods implemented are Methods 3500, "Organic Extraction and Sample Preparation," Method 3541, "Soxhlet Extration," Method 3600, "Clean-up," Method 3620, "Florisil Column Clean-up," Method 8000, "Gas Chromatography," and Method 8082, "Polychlorinated Biphenyls by Capillary Gas Chromatography."

A total of 234 field split samples were received from September 19, 1997, to November 13, 1997. The samples were also sent to a commercial laboratory; the results reported by the commercial laboratory will be used in the remedial investigation report of the David Witherspoon 1630 Site. A total of 340 extracts were processed through the system, including 26 method blanks, 29 blind quality control (QC) samples, and 24 matrix spike/spike duplicates. The samples were processed during 24 working days, averaging 9.8 extractions per day. A total of 807 extracts were processed through the gas chromatograph and data interpretation module (DIM) SLMs, which included 19 calibration standards, 111 daily calibration check standards, and 337 dilutions and reruns of the original extracts. PCBs were detected in more than $60 \%$ of the soil samples; this is uncharacteristically high for a typical remediation effort.

The chemical performance of the automated PCB laboratory was evaluated according to the QC measures specified in SW-846, Chapter One, Sect. 4.4, "Laboratory QA and QC Procedures" as well as specific criteria listed in the methods noted previously. Performance parameters included:

- $\quad$ blind QC standards, 
- method blanks,

- daily calibration check standards,

- matrix spike and matrix spike duplicates,

- surrogate recovery,

- $\quad$ field duplicate samples,

- method detection limits, and

- results data reported by the commercial laboratory.

The CAA system met or exceeded all the laboratory quality assurance (QA) and QC requirements. Early in the project, the contractor performing the remedial investigation recognized the quality of the CAA data and used the CAA data as a quality check on the contract laboratory. Normally, this quality check task is performed by sending about 10 to $15 \%$ of the samples, as field splits, to a second laboratory.

Histograms of the PCB concentration in the samples analyzed by the CAA system and the commercial laboratory are shown in Fig. 1. The two distributions are essentially identical. Statistical assessment of the two data sets reveals no statistical difference between them.

A post-Witherspoon project review of chromatograms processed through the DIM suggests that the DIM may save approximately $50 \%$ of the chemist's effort devoted to review of raw data. The DIM may also yield fewer false negative results, which is an important attribute from the environmental remediation and public health perspectives.

Work flow and task time data for the CAA system were collected during the Witherspoon project. From this information, estimates were made of potential savings in the per-sample analysis cost achievable through the implementation of the CAA. The analysis was performed on the basis of labor and material only. Assumptions made in this analysis include:

- implementation of a software system to electronically manage the data and automatically generate the data package (System Mods),

- limited operator attention to the system (because of safety considerations, the CAA team required use of the "buddy" system during the entire Witherspoon project),

- operation of the system while unattended on a second shift, and

- configuration of the system to automatically dilute and rerun extracts.

The results of the cost projections are summarized in Fig. 2. The projections suggest a per-sample analysis cost of less than $\$ 50$. The commercial laboratory per-sample contract price for the Witherspoon project was $\$ 82$. 


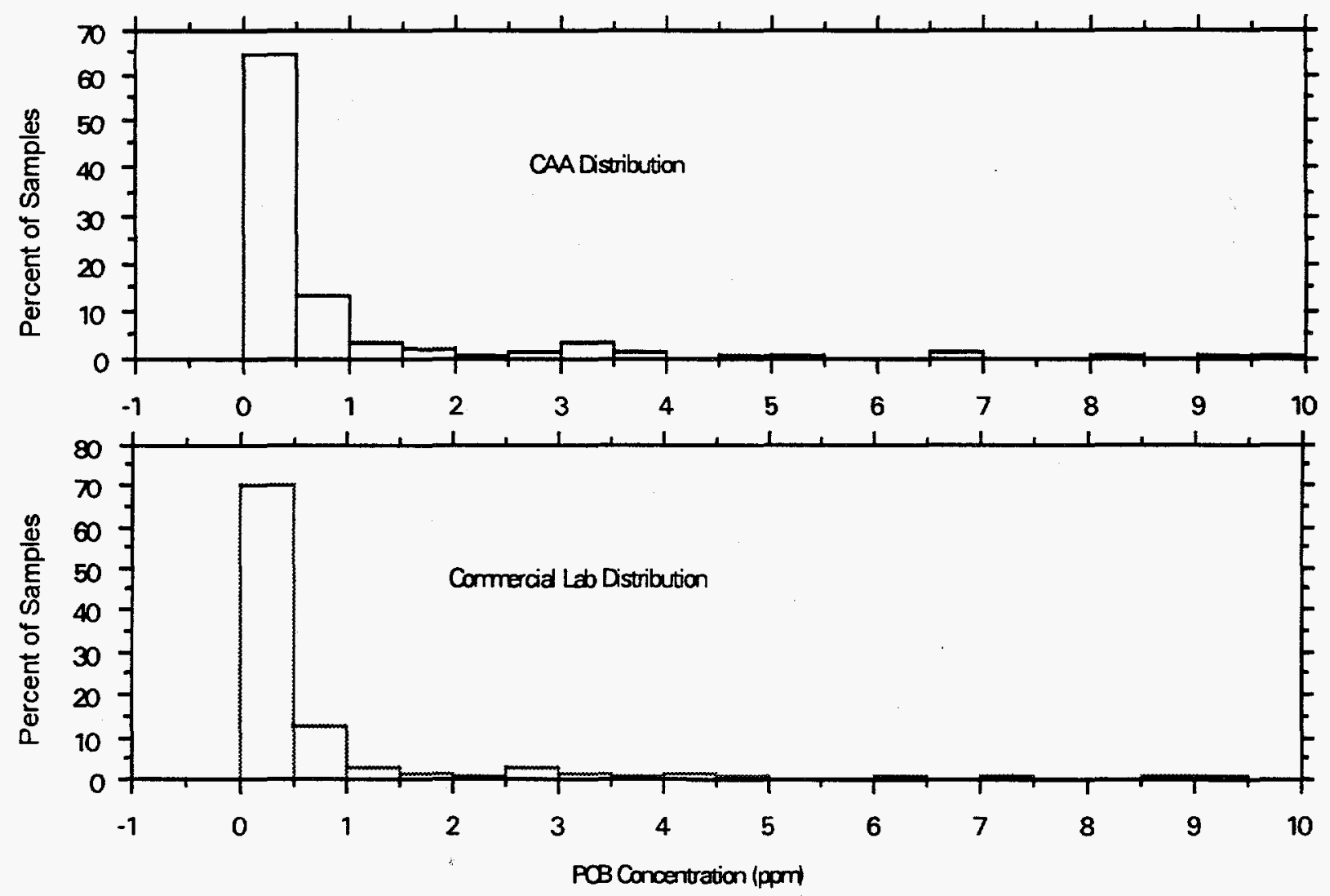

Fig. 1. Histogram distribution of PCBs in the Witherspoon soil samples.

The Witherspoon project identified areas needing further development. These include:

- electronic management of data,

- making the $\mathrm{HCI}$ more intuitive to the laboratory personnel, and

- implementing a simple decision-making capability in the TSC.

Participation in the Witherspoon project was an extremely valuable learning experience for the CAA development team. The project team learned that most of the elements of the CAA laboratory are robust. The chemists reported that they never had processed that many samples in such a short time frame with so few equipment problems. Only four samples $(\sim 0.3 \%$ of the total number of extractions) were lost because of system errors while being processed. In these cases the manual sample preparation steps were repeated and the samples resubmitted to the system. The project team also learned that greater attention needs to be paid to the needs and the perceptions of the chemist. 


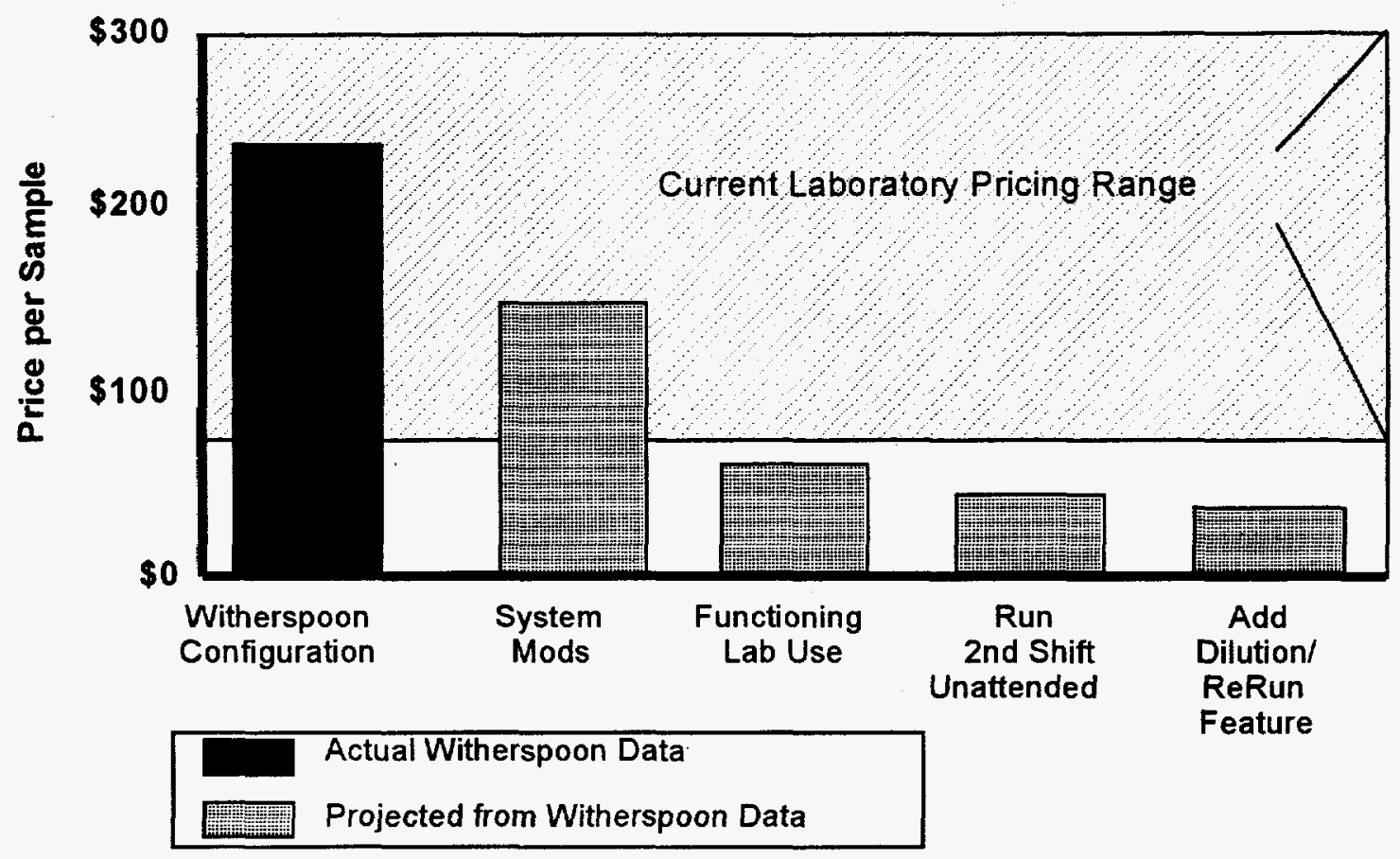

Fig. 2. CAA cost comparison projections. 


\begin{abstract}
The Chemical Analysis Automation (CAA) project is developing a standardized modular automation strategy for automated chemical analysis. In this automation concept, analytical chemistry is performed with modular building blocks that correspond to individual elements of the steps comprising the total analytical protocol. With a standardized set of behaviors and interactions, these blocks can be assembled in a plug-and-play manner into an integrated automation system. The first fully automated CAA system is designed for the determination of polychlorinated biphenyls (PCBs) in soils. This system was used as a technology demonstration and development testing activity in conjunction with the surface soil characterization activities at the David Witherspoon 1630 Site. The automation system consisted of five hardware standard laboratory modules (SLMs), one software SLM, a task sequence controller, and the human-computer interface. The demonstration and development testing were conducted and evaluated against the benchmark methods defined by the U.S. Environmental Protection Agency (EPA) "Test Methods for Evaluating Solid Waste, Physical/Chemical Methods, SW-846" manual. A total of 234 field split soil samples were processed through the system in 24 working days. PCBs were detected in more than $60 \%$ of the samples. The output of the demonstration was regulatory compliant data packages. The CAA system met or exceeded all laboratory quality assurance and control requirements defined by the EPA. A statistical analysis of the results obtained with the CAA automated laboratory and those reported by the commercial laboratory revealed that there was not a statistical difference between the two data sets. Based on work flow and task time information collected during the Witherspoon project, we estimate that the automation system can yield a per-sample analysis cost of less than $\$ 50$. This report presents details of the data obtained from the technology demonstration and development testing; the chemical, hardware and software performance; and areas needing further development.
\end{abstract}




\section{INTRODUCTION}

The Chemical Analysis Automation (CAA) project is a multi-institutional project. Collaborators consist of four U.S. Department of Energy (DOE) laboratories-Idaho National Engineering and Environmental Laboratory, Los Alamos National Laboratory (LANL), Oak Ridge National Laboratory, and Sandia National Laboratories, two universities-the University of Florida, Gainsville, and the University of Tennessee, Knoxville; and a technology commercialization partner, Advanced Power Technologies, Inc. The purpose of the CAA project is to develop standardized, software-driven, site-deployable robotic laboratory systems that will lower the per-sample analysis cost, decrease sample turnaround time, and minimize human exposure to hazardous and radioactive materials associated with the DOE environmental remediation activities.

The first integrated robotic laboratory system developed by the CAA project is a system designed for the determination of polychlorinated biphenyls (PCBs) in soil matrices. The CAA project designates this system as the PCB standard analysis method (SAM). The PCB SAM is fully compliant with the regulatory requirements specified in the Resource Conservation and Recovery Act "Test Methods for Evaluating Solid Waste, Physical/Chemical Methods," EPA Publication SW-846. ${ }^{1}$ The first phase of development testing of the PCB SAM was completed in the Summer of 1997. Final development testing was conducted in conjunction with the DOE site characterization study at the David Witherspoon, Inc. 1630 Site.

The DWI Landfill 1630 Site is located at 1630 Maryville Pike in Knox County, Tennessee. The landfill was unregulated from the 1950s until its closure in 1974. The site is located within a residential and commercial mixed-use area. Solid and liquid wastes from salvage and industrial operations were disposed of in the landfill. These wastes include PCBs and low-level radioactive contaminated metal. In 1994 the Tennessee Department of Environment and Conservation and DOE entered into a consent order for the remediation of the site. The DWI 1630 Site is a state of Tennessee Superfund site, and remediation is being conducted under the Tennessee Hazardous Substance Remedial Action Program. In accordance with the consent order, DOE is conducting a remedial investigation at the DWI 1630 Site to determine whether hazardous substances present at the site pose a hazard to human health and the environment. Numerous activities are scheduled as part of the current remedial investigation and are described in detail elsewhere. ${ }^{2}$

Activities of interest to the CAA project involve the collection of surface soil samples and the determination of their PCB content. This report describes the results of the development testing of the PCB SAM that was conducted using field split samples obtained from the surface soil sampling element of the remedial investigation.

\footnotetext{
1 "Test Methods for Evaluating Solid Waste, Physical/Chemical Methods," EPA Publication SW-846, 3rd ed., U.S. Environmental Protection Agency, 401 M Street, SW, Washington, DC 20460.

2 "Work Plan for the Remedial Investigation for the David Witherspoon, Inc., Landfill 1630 Site, Knoxville, Tennessee," DOE/OR/02-1548\&D1, March 1997.
} 


\section{DESCRIPTION OF THE CHEMICAL ANALYSIS AUTOMATION PARADIGM}

The CAA project views the chemical analysis laboratory as a multi-step process that transforms a sample into information. Each step within the process receives an input and generates an output that becomes the input for the next step. The initial step involves receiving the samples, logging them into the Laboratory Information Management System (LIMS), and producing a "worksheet" that defines the process or processes to be performed by the laboratory. The final step involves a quality assurance specialist receiving a draft data package for review and approving it for release to the laboratory's client.

Viewing the chemical analysis laboratory from this perspective requires the development of the total set of relationships among the components. This includes an identification of the components, the functional position and relationship of each component within the entire process, how each component functions, and the component behavior that is necessary for operation in an integrated system.

Every analytical method contains three basic steps: sample preparation, analyte measurement, and data interpretation. The initial component, sample preparation, remains time consuming, labor intensive, potentially hazardous to personnel, and a major source of errors. Automation of the second component, analyte measurement, has received the most attention to date, with many commercial vendors providing sophisticated, automated instruments. The final component, data interpretation, which includes data processing, reporting, and quality assurance, consumes about $50 \%$ of the staff resources in environmental laboratories. Furthermore, data interpretation tasks require the expertise of an experienced analyst. Thus, the first and last components present the greatest opportunities for the CAA program to improve automated methods.

In addition to focusing on the automation of sample preparation and data interpretation, the CAA program addresses the greater challenge of combining automated sample preparation and data interpretation components with measurement instruments to form totally integrated systems. At present, many individual pieces of equipment that perform sample preparation and analyte measurement have been automated by instrument vendors, but the integration required for a totally automated, multivendor system remains a difficult task. Lack of integration standards requires each system to be custom-built by either the vendor, the customer, or a third-party system integrator. To achieve its goal of developing automated laboratory systems, the CAA program is developing and demonstrating the implementation of hardware and software standards for system integration. Laboratory automation should be analogous to office automation, where standard hardware interfaces and software drivers facilitate the plug-and-play integration of computers, printers, modems, CD-ROMs, and other components into efficient, automated systems.

The plug-and-play concept overcomes problems that have hindered automation of environmental analysis. Diverse matrices, such as soil and water, do not require separate automated systems. Rather, the replacement of a sonication or Soxhlet extraction step (for extracting analytes from soil samples) with a liquid-liquid extraction step (for removing analytes from aqueous samples) allows the same system to process both sample matrices. The method that is attached to a particular sample defines which steps are performed. Similarly, the introduction of a procedure, such as Florisil ${ }^{\mathrm{TM}}$ cleanup of soil samples contaminated with PCBs, can be accomplished by plugging a Florisil ${ }^{\mathbf{T M}}$ column component into the automated system. The system's master controller identifies the new component, reconfigures the system, and manages the flow of samples through all components. 
The CAA laboratory automation concept involves three key concepts: laboratory unit operation (LUO), standard laboratory module (SLM), and SAM. The LUO is an operation or several operations performed by an automation module that does not require intervention by either a human or a robot external to the automation module. An SLM is a logical combination of LUOs that, when linked together, performs a complete subtask within an analytical protocol. SLMs have several attributes including:

- An SLM can be either hardware or software.

- SLMs conform to a well-defined interface standard.

- An SLM expects to interact with a supervisory control system for integrated operation.

- An SLM can function independently in stand-alone operation.

- SLMs can be combined with other SLMs to form systems of greater complexity.

A SAM is a fully integrated sample analysis system, consisting of software and hardware SLMs, which automates the complete analysis process from sample input through interpreted data as the output. SAMs have several attributes including:

- The SAM controller orchestrates all activities, including the management of data.

- The user operates and monitors the SAM from an intuitive human-computer interface.

- The processing of samples is determined by the method attached to the sample.

- Samples associated with different methods can be processed concurrently.

- Temporary unavailability of an SLM merely pauses the method.

- The SAM has robust error recovery capability.

A block diagram of the CAA automated laboratory is shown in Fig. 2.1.

The basic building block of the CAA system is the SLM. Each SLM performs a group of LUOs required by an analytical procedure. An embedded microprocessor on the SLM is programmed to direct the execution of LUOs. For example, a Soxhlet SLM, used to extract analytes from solid samples, contains liquid-handling and heating LUOs that are controlled by the SLM's embedded microprocessor. SLMs communicate with the system controller, known as the master controller or task sequence controller (TSC), through standard hardware and software communication interfaces, which can be either serial (RS232 or Ethernet) or parallel general-purpose interface bus (GPIB or IEEE-488). The software interface consists of a set of vendor-defined commands and events that are recognized by the SLM. A standard set of responses to commands allows the master controller to monitor the actions of the SLM.

Once the sample is delivered to an SLM in the ready state and processing is initiated, the SLM requires no further interaction with any system component, including the master controller, to process the sample. When processing is complete, the SLM notifies the master controller. If the SLM malfunctions while processing a sample, it must inform the master controller of the malfunction without being queried. The SLM must be able to respond to queries from the master controller at all times.

Variations of SLMs that can be interfaced to the master controller include: analytical instrument modules (AIMs), data interpretation modules (DIMs), and service support modules (SSMs). These SLM variations, although referred to by different names, possess the attributes of SLMs and meet the SLM interface and communication requirements. SLMs handle tasks associated with sample preparation (e.g., sonication, extraction, filtering, concentration, dilution, separation, and dissolution), AIMs perform measurements, DIMs interpret data, and SSMs, typically robots, transfer physical items. The robot SLM is 
the only SLM that can enter the space of another SLM to transfer samples and consumables. However, one SLM may not interact directly with another SLM.

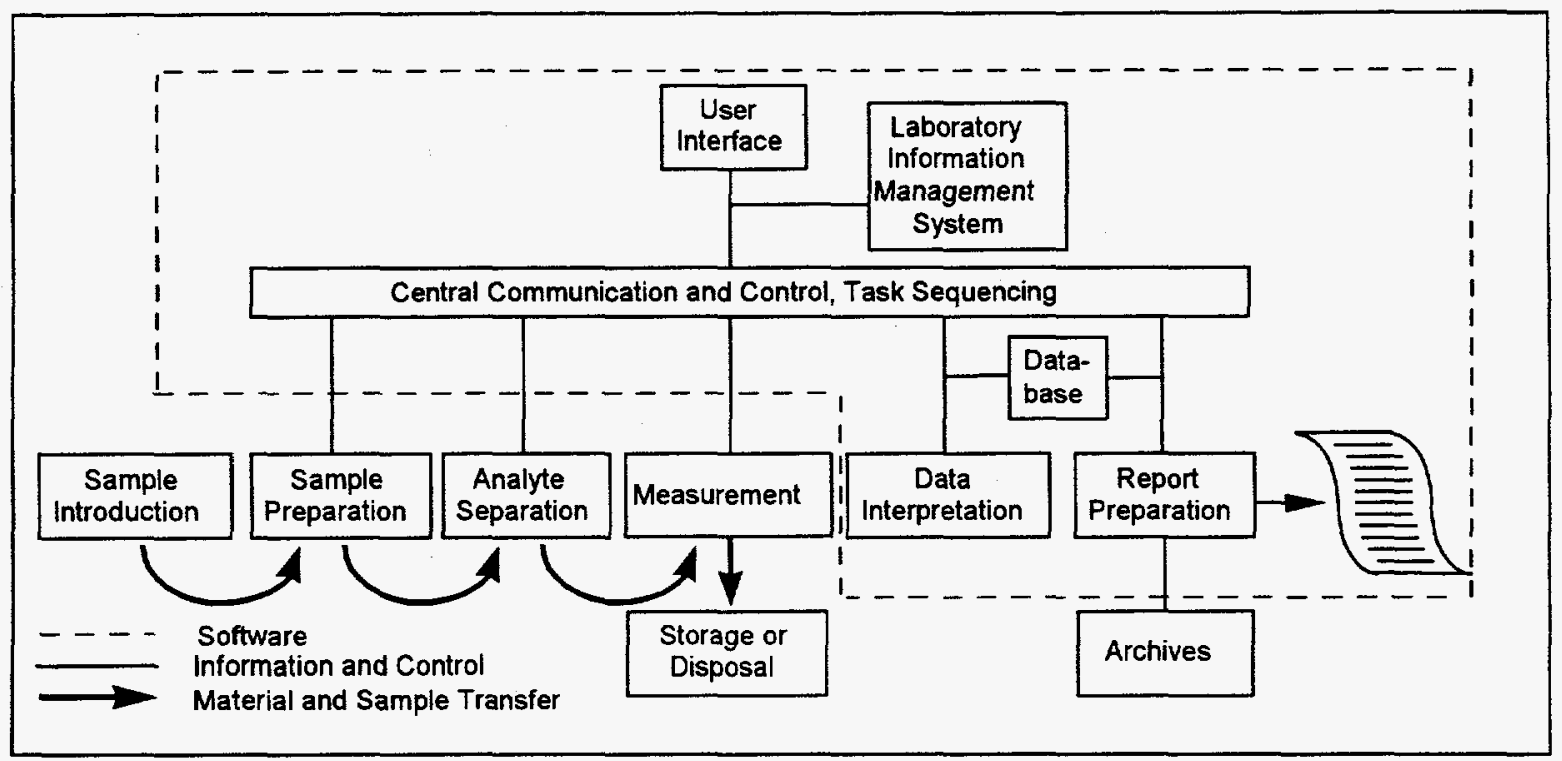

Fig. 2.1. Block diagram representation of CAA paradigm.

A data manager conducts all database functions. The data manger is a SLM. The CAA database contains the detailed information needed for the operation of the SAM and is the repository for information generated while the SAM is processing samples. Because of the wide variety of Laboratory Information Management Systems (LIMS) in existence, communication with the LIMS occurs through a SLM style interface.

Sample transfers among the SLMs are handled by robot under the direction of the master controller. Although AIMs have embedded computers and the required hardware ports to interface with the master controller, software additions are required for the AIMs to interact with the master controller. The DIM is a software module that interprets data from an AIM using a combination of chemometric methods. Each analytical method has a unique DIM. In addition to the master controller and modules described previously, two other components are required to complete a system: a human-computer interface (HCI) and a dedicated database. The HCI is used by the operator to enter and track samples, monitor the system, maintain the modules, and access the database. The database contains information on analytical methods, maintenance procedures, module capabilities, samples and sample processing, raw and processed data, operator qualifications, and final results. Audit trails and processing diaries for each sample are also stored in the database. The database can transfer information to the LIMS. The report generation function can be implemented either by the database or by the LIMS. Fully automated systems are capable of performing standard methods of analysis. The configuration of components and directions for each procedure are defined in a SAM. When the operator uses the HCI to enter a sample into the system, a SAM is attached to the sample. The master controller checks the system configuration and retrieves the information required to perform the SAM from the database.

The CAA project realizes that in the near term many laboratories may not be able to justify the cost of a totally automated system regardless of its flexibility. With this in mind, a path to fully automated 
systems exists that allows the purchase of individual SLMs to operate in a stand-alone mode. Such SLMs can alleviate bottlenecks in sample processing; their embedded microprocessors, keypads, and displays permit direct interaction by operators. Since SLMs are built to standard specifications, they have the capability to become components of larger more automated systems. A mini-SAM represents an intermediate mode of integration between the stand-alone SLMs and fully automated systems that can perform all tasks associated with a SAM. The mini-SAM is a group of two or more SLMs that perform several steps within a total method. A mini-SAM might include extraction and concentration SLMs or a gas chromatograph AIM and a PCB DIM. A technician or a robot can perform sample and consumable transport.

There are no specific dimensions for an SLM. Most SLMs are designed to operate in a standard laboratory hood and for compatibility with this environment. All interface connections (including those for utilities, gases, and liquids) are located on the front or rear panels. Sample container input and output ports are located on the front panels and are accessible to a robot. Compressed gases common to laboratories (e.g., nitrogen, helium, and filtered, dehumidified, compressed air) are supplied from a source external to the SLM. Because many laboratories do not provide common vacuum lines, SLMs requiring vacuum must contain their own vacuum systems. Liquid solvents for a fully integrated system are supplied from a common reservoir.

All SLMs must allow transfer of samples to other SLMs as specified by the analysis method. The robotic SSM performs container transfers. The robot module handles vials and beakers of varying sizes and configurations. Because of the different sample sizes encountered in an integrated system, it is not possible to have a rigid standard for container sizes. Placing multiple robot hands on an automated hand change station accommodates differences in container sizes. Container transfer is relatively simple and can take advantage of queuing, which is accomplished by placing racks on the automation bench. 


\section{DESCRIPTION OF THE POLYCHLORINATED BIPHENYL STANDARD ANALYSIS METHOD}

\subsection{INDIVIDUAL STANDARD LABORATORY MODULES}

The hardware SLMs tested included a four-channel Soxhlet extractor (CAA design), a high-volume concentrator (CAA design), a cleanup column module, and a gas chromatograph (GC) equipped with an electron capture detector (ECD) and a liquid autosampler. Samples are transported among the SLMs by a robot moving along a linear track. The software modules tested included the PCB DIM, TSC, and the $\mathrm{HCI}$, all created by the CAA software development team.

\subsubsection{Soxhlet Extractor Standard Laboratory Module}

The automated Soxhlet SLM performs the identical process with four separate extractors operating in parallel. The analyst weighs and dries a 10-g soil sample and places it in a cellulose thimble with a metal ring attached to the opening at the top. The robot inserts the thimble into one of the SLM's four input ports. The SLM uses a video imaging system to confirm that a thimble is indeed present. A magnetic ring is lowered, which picks up the thimble. The SLM then retracts the thimble into the condensing/refluxing column. A clean empty beaker is placed on the heating mantle, again by the robot, and once the vision system confirms the presence of the beaker, the heating mantle and beaker rise automatically until the beaker contacts the bottom of the condensing/refluxing column. At this point, the SLM needs no further analyst or robot intervention until the extraction is complete. The SLM dispenses a preset volume of organic solvent through a supply valve at the top of the condensing/refluxing column; the solvent flows down the column through the thimble and into the beaker. Power is supplied to the heater, which is controlled by the SLM, until the solvent reaches its boiling point. The SLM then lowers the thimble into the boiling solvent, where it remains for approximately 60 minutes. After the allotted time, the SLM lifts the thimble out of the boiling solvent for the solvent refluxing period. When refluxing is complete, the reflux valve closes, collecting condensing fluid in the column, reducing the volume of solvent in the beaker, and concentrating the analytes in the remaining liquid. The SLM checks the solvent level and, if the level drops below a specified valve, the SLM opens the reflux valve momentarily to allow additional solvent back into the beaker. Once the extraction is complete and the beaker has had time to cool, the SLM lowers the beaker and sends a signal indicating that the process is complete. The robot removes the beaker containing the extracted analytes, discards the thimble containing the original soil sample, and the process is ready to begin again with a new soil sample.

\subsubsection{High-Volume Concentrator Standard Laboratory Module}

The high-volume concentrator (HVC) SLM automates the Kuderna-Danish evaporation process typically used by environmental laboratories to reduce the volume of the extraction solvent. The robot takes the beaker from the Soxhlet SLM port and places it in the beaker port of the HVC SLM. A glass output vial, capped with a septum, is provided to the second port. Once the beaker and vial are in place, no further action by the robot or analyst is needed until the process is complete. A sensor confirms the presence of the beaker and that it is an allowed size. The SLM lowers a metal tube into the beaker and siphons the solution into the boiling chamber. Clean solvent is sprayed onto the sides of the beaker to rinse any residual analyte to the bottom, and this rinsate is added to the boiling chamber.

The SLM applies a selectable partial vacuum to the boiling chamber and applies heat. Heating preferentially boils off the solvent, leaving the analytes behind. Partial refluxing increases the effectiveness 
of separation. The vapor that passes out of the refluxing column is collected in a waste solvent container for reclamation or disposal. Solvent exchange may be effected at this point by adding a different solvent and repeating the process; however, the current PCB analysis does not require a solvent exchange. Sensors determine when the solvent volume is sufficiently reduced, and the concentrate is then transferred to another chamber for final volume adjustment. Rinses of the boiling chamber are also transferred, ensuring complete transfer of the analytes of interest. If the final volume needs to be increased, the SLM adds solvent until sensors indicate that the exact volume has been reached. If the final volume needs to be reduced, the SLM evaporates solvent by blowing pure nitrogen against the solvent surface until the proper volume is reached. The SLM is precalibrated to yield the final volume desired for the analysis. The current version of the $\mathrm{HVC}$ can provide either a $1-\mathrm{mL}$ or a $10-\mathrm{mL}$ final volume.

In the output port, the SLM inserts a needle through the septum covering the output vial, partially evacuates the vial volume, and then transfers the volume-adjusted extract through the needle into the vial. The needle is extracted, and the SLM signals that the process is complete and that the vial is ready for pickup. Once the vial and input beaker are removed, the concentrator SLM is ready for the next sample.

\subsubsection{Column Standard Laboratory Module}

The column SLM automates the extract cleanup process using traditional solid-phase adsorption technology. The SLM is a modified version of a Gilson Sampling Injector and Syringe Pump apparatus. The robot inserts the capped glass vial containing the sample extract into the SLM input port; a septum sealed glass output vial; a fresh, prepacked column; and a clean empty uncapped collection vial to receive the eluant from the column. The SLM requires no further attention from the robot until the cleanup process is complete. The column SLM uses the single syringe and valve configuration of the Gilson Syringe Pump as a low-pressure pump for transferring liquids. The SLM uses a three-port valve to allow aspiration from the reservoir port or the needle port. Dispensing occurs only through the needle port. The sampling injector is equipped with a rinsing station to clean the needle externally and internally. Since the cleanup columns are prepacked with a solid adsorbent material, the cleanup method is determined by which column is presented to the SLM. The PCB SAM uses prepacked Florisil ${ }^{\mathrm{TM}}$ columns. For PCB analysis, the analytes of interest are not retained by the adsorbent; therefore, no additional solvents are added, and the concentration of the analytes is not changed. If different fractions containing different classes of analytes were desired, those samples could be eluted with additional solvents and be returned to the HVC for volume adjustment. The SLM uses the needle to penetrate the septum sealing the input vial and withdraws most of the solvent extract. This is transferred to the cleanup column, is eluted by gravity with solvent, and is then collected and transferred to the collection vial. Any excess extract is discarded to a waste container. A predetermined volume, typically $2 \mathrm{~mL}$, of the purified extract is removed from the collection vial and transferred to the output vial. The output vial is then ready for pickup by the robot. Once all the vials and the used cleanup column are removed, the SLM signals that it is ready to process another sample.

\subsubsection{Gas Chromatograph Standard Laboratory Module}

Most current GCs are equipped with automatic injectors. In addition, almost all GCs have some means of connecting the detector output to a data acquisition device. Therefore, modern GCs need very little modification to become SLMs. The current GC SLM, for example, is an off-the-shelf model with software additions required to make it a compliant SLM.

The GC SLM operation begins when the robot places a septum-capped vial in the GC autoinjector tray. Upon receiving a "start" command from the TSC or the analyst, the autoinjector pierces the septum with a syringe needle and withdraws a small amount of the sample extract, typically $2 \mu \mathrm{L}$, into the syringe 
barrel. The syringe is positioned over the injection port of the GC and injects the sample. The SLM then rinses the syringe with clean solvent. After the GC run is completed, the GC data station software locates and integrates the area of the elution peaks using the parameters defined within the $\mathrm{GC}$ data station software. The resulting raw chromatogram and peak data are assembled into a standard file format and are transferred to a shared disk area where they can be accessed by the DIM. Once the robot removes the vial from the autoinjector tray after the GC run is completed, the GC SLM signals that it is ready for the next sample.

\subsubsection{Robot Standard Laboratory Module}

The robot SLM transports materials needed in the chemical operations of the PCB SAM. The TSC instructs, via its transport command, the robot SLM to move an item from one location to another. The transport command includes parameters describing the type of item being transported, the SLM, port, and port index that the item should be retrieved from or delivered to. The robot changes grippers to accommodate the different sizes of objects used in the PCB SAM.

\subsubsection{Task Sequence Controller}

The TSC is a software program that provides the scheduling, management, and coordination of all system SLM activities. From a software perspective, each analytical method is described by a "script" or list of operations that make up the method. The TSC executes scripts by expanding them into the set of elemental operations to be performed by SLMs contained in a particular SAM.

The TSC selects an SLM to perform each operation based on the availability of the SLM, sample processing priorities, and any device selection criteria specified in the script. Selection of the SLM to perform an operation is made when the TSC interprets the script command specifying the resource. Thus, redundant SLMs may be added to the system at any time to increase sample throughput. Similarly, if an SLM is taken off-line or reports an error, the TSC will route samples around it. If an SLM is off-line and a redundant module is not available the TSC places the samples into the SLM queue rack. When the SLM becomes available the TSC automatically resumes the sample processing. It is the responsibility of the TSC to ensure that all material transport is performed for the selected SLM and to initiate the operation of each SLM.

The TSC is able to communicate with any compliant SLM. The SLM vendor defines the commands necessary to operate the SLM. Responses to commands issued by the TSC must follow a prescribed format. Common communication protocols for all commands are less challenging than might first appear because the SLMs are highly autonomous and only minimal amounts of information must be exchanged between the TSC and the SLM.

The TSC is a software program that gives commands to SLMs in a format that an analyst need not understand. The HCI provides an intuitive interface between the analyst and the TSC. The analyst understands the laboratory operation in terms of analytical methods and laboratory procedures. The $\mathrm{HCI}$ allows the analyst to use a graphical user interface to submit samples and prescribe required laboratory procedures, plus any special sample priority, in a way that is easily understood by an analyst. Then for actual execution, the $\mathrm{HCl}$ translates these commands into instructions that are recognized by the TSC. Similarly, messages from the SLMs to the TSC, for example ready conditions or error conditions, are passed on to the $\mathrm{HCl}$, which translates them for the analyst into terms of laboratory procedures. 


\subsubsection{Data Interpretation Module}

The DIM automates the analysis of data from the analytical instrument. It also performs quality assurance and quality control calculations. Although the DIM is a software tool, it behaves like any other SLM, using the same communication protocols to receive commands from the TSC and to provide responses to the TSC. The TSC initiates the analysis of a sample by reading the script containing the commands that describe the analytical method. Information collected during sample preparation that will be required later by the DIM is written to a database. When the analytical instrument SLM has produced the raw data, the data file is passed to the DIM to begin data analysis.

The DIM determines the presence of analytes of interest and their concentration. The analytical method used by the DIM will depend on the analytical instrument used and the analytes of interest. Analysis of metals by atomic absorption requires simple univariant analyses; however, analysis of gas chromatograms for PCBs requires complex, parallel, multivariant analyses with variable weighting values.

The DIM writes the results of the analysis in any of a variety of formats. It will send the data in the format required by the system database but can also, for example, write the data in a form readable by common spreadsheet programs. Data retrieved from the database can be examined and reanalyzed by the operator using the DIM's off-line operational mode.

\subsection{INTEGRATED SYSTEM}

When hardware and software modules are assembled together to perform a complete laboratory analysis [for example, a designated U.S. Environmental Protection Agency (EPA) method], the integrated system is called a SAM. This naming convention reflects the CAA project's design objective of standardized, modular equipment that can be used either as stand-alone equipment or be easily integrated with one another. The modularity of the SLMs and the use of a common communications protocol allow SLMs to be arranged in a variety of different combinations.

The functionality of a SAM can be provided by different sets of SLMs, e.g., a sonication SLM could replace a Soxhlet SLM while retaining the ability to determine PCBs in soil matrices. Also, a particular set of SLMs can in fact represent multiple SAMs. For example, adding a gas chromatograph mass spectrometer (GC-MS) SLM to a SAM containing a GC equipped with an electron capture detector (ECD) provides at least three possible SAMs: (1) a SAM applicable to the determination of PCBs, (2) a SAM applicable to the determination of chlorinated pesticides, and (3) a SAM applicable to the determination of semivolatile compounds. The definition of the SAM is specific to the sample, and the SAM definition occurs when the sample and the TSC script are associated with each other. It is also important to note that samples being processed by all the SAMs can occur concurrently.

A PCB SAM using a GC equipped with an ECD was used for the Witherspoon project. The PCB SAM is configured to meet the requirements specified in EPA SW-846 Methods 3541, 3620A, and 8082 for the analysis of PCBs in soils. A graphical representation of the PCB SAM is shown in Fig. 3.1.

Figure 3.2 is a photograph of the PCB SAM as configured for the Witherspoon development test. The entire assembly is contained in a vented enclosure to minimize exposure of the operating staff to solvent vapors. The SLMs are arranged along one side of the robot rail. Because the availability of a resource for the next stage of sample processing cannot be ensured when the sample is ready, racks are used to queue items between each SLM. Consumables are placed in racks. The robot tool stand is required 
to allow the robot to handle the differently sized items used in the SAM. When the sample is queued in a rack, it is covered to reduce the possibility of contamination.

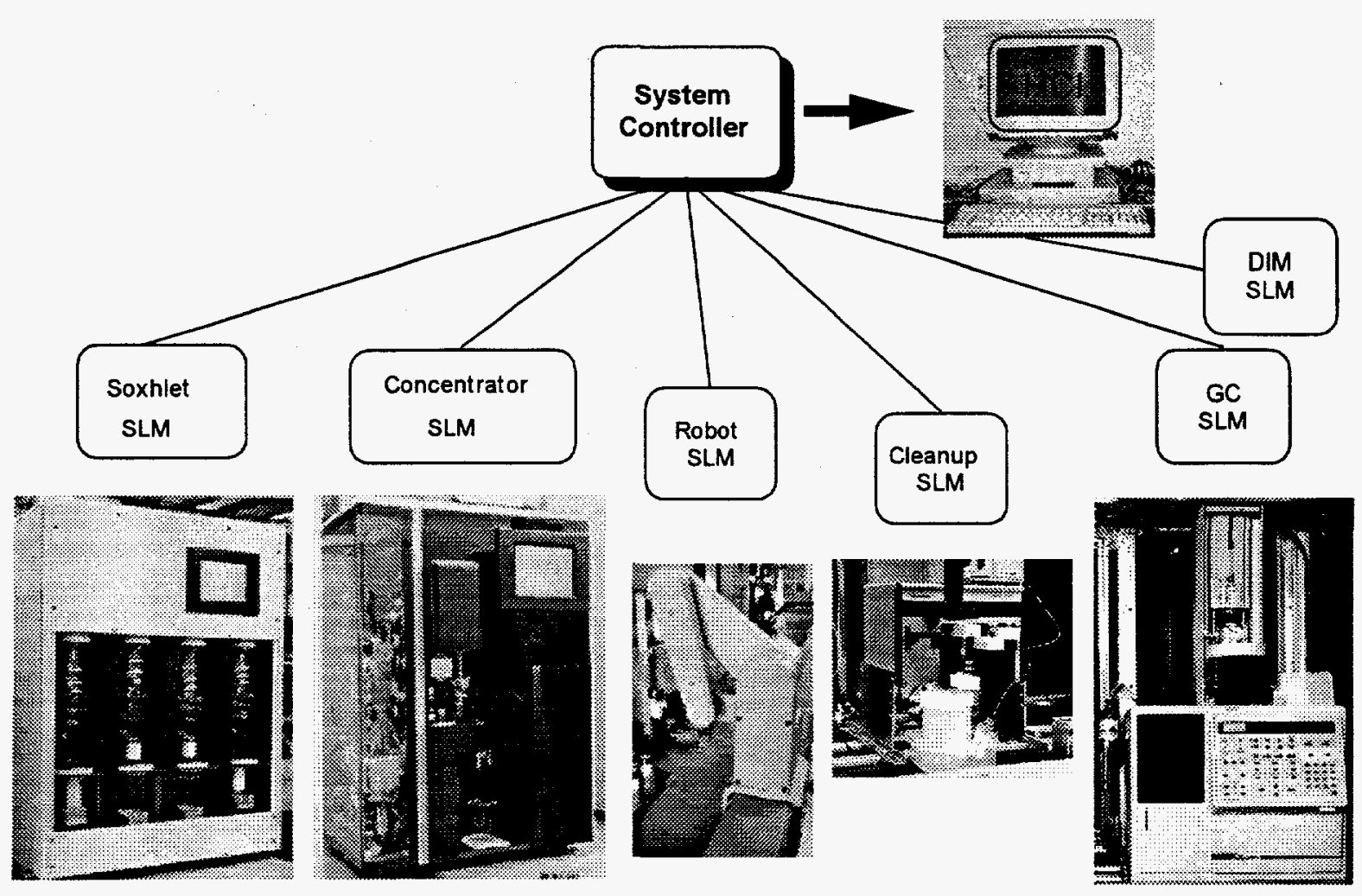

Fig. 3.1. Graphical representation of the PCB SAM.

\subsubsection{Role of the Analyst}

The first steps of sample preparation are performed by the analyst. The sample is logged in, and a portion is set aside for drying to determine percent moisture content. An aliquot used for analysis is removed, weighed, mixed with anhydrous sodium sulfate, and ground to a powder. The analyst may note that the sample requires additional special handling, for example, removing bits of debris. The analyst then adds whatever surrogates, spikes, or other chemicals that are required for a particular sample. Racks containing the consumables such as the beakers, vials, and cleanup columns used by the various SLMs must also be prepared by the analyst. The analyst must also ensure that adequate quantities of solvents and gases are available to the SLMs.

The analyst weighs the sample into a prerinsed cellulose thimble and places the thimble into the sample queue rack for the Soxhlet SLM, repeating this for each sample in the batch. The analyst enters the sample information into the system through the HCI, identifying the required protocol and any special priority status. The position of the sample in the rack is used by the automated equipment to initially identify each sample. Subsequent tracking of the samples is handled automatically by the TSC. The 
analysis can now begin, and, under normal conditions, no further intervention is required until the batch of samples is completed.

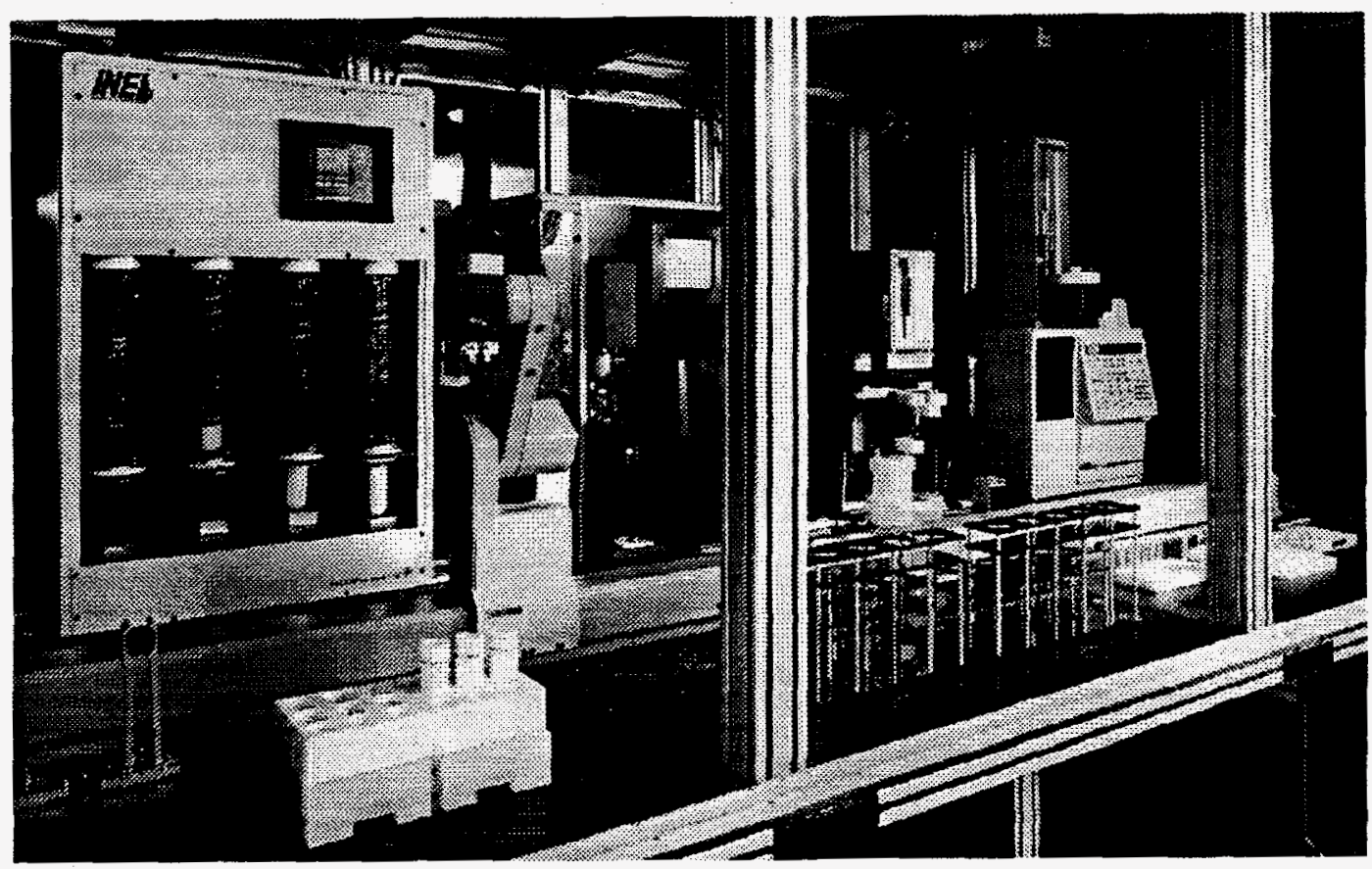

Fig. 3.2. PCB SAM configuration for the Witherspoon test.

\subsubsection{Role of the Standard Analysis Method}

The operator can give a "start" command for a single sample or a whole batch. The TSC executes the appropriate script for the sample to determine the sequence of steps. For this particular PCB SAM, the script calls for the sample to be introduced into the Soxhlet SLM. The TSC instructs the robot to retrieve the sample from the sample queue rack and move it to the input port of the Soxhlet SLM, after determining the Soxhlet is ready to receive a sample. The robot also brings the consumables to the SLM. When the SLM indicates to the TSC that these articles are present, the TSC sends the SLM a start command and the SLM extracts the sample as described previously.

When the SLM completes the sample processing, it informs the TSC via a "complete" response. The TSC then instructs the robot to execute the appropriate transport commands to remove the processed sample and the used consumables from the SLM. The sample is then ready for the next processing step; the SLM is ready to receive and process the next sample. 


\section{PROCEDURES}

\subsection{CHEMICAL PROCEDURES}

Chemical performance of the CAA systems is being evaluated against benchmark methods as defined by the EPA. The EPA methods, detailed in "Test Methods for Evaluating Solid Waste, Physical Chemical Methods, SW-846,"] prescribe system parameters for each component of the analytical analysis platform. In addition to the system parameters, SW-846 provides protocols for instrument calibration, sample preparation, and quality control.

The analytical platform consists of five separate chemical functions: sample preparation, extraction, concentration, cleanup, and detection. The corresponding EPA SW-846 methods are 3541, 3620 , and 8082 .

SW-846 Method 3541 identifies sample preparation procedures and target analyte removal by Soxhlet extraction. Ten grams of soil is mixed with an equivalent amount of sodium sulfate and is placed in a cellulose extraction thimble. The thimble is placed in boiling solvent housed inside a collection beaker. The solvent, as suggested in the SW-846 method, is a mixture of 1:1 acetone and hexane. During the extraction phase, the thimble containing the sample is immersed in the boiling solvent for an hour. After the 1-hour extraction, the thimble is raised out of the boiling solvent, allowing the condensed solvent to rinse the constituents of interest from the solid matrix. The Soxhlet SLM has the capability of performing four simultaneous extractions.

Following extraction, the collection beaker is automatically transferred from the Soxhlet SLM to the HVC SLM. Boiling and evaporation of the extract solvent accomplish the solvent volume reduction in the HVC. A combination of solvent addition and nitrogen blow-down are used to make the final volume adjustment. Optical sensors control the final volume adjustment. The system is calibrated to yield the specific liquid volumes.

EPA Method 3620 discusses the use of Florisil ${ }^{\text {TM }}$ columns as a cleanup procedure prior to sample analysis by gas chromatography. The active ingredient is magnesium silicate, which possesses acidic properties. To effect cleanup, the sample must be dissolved in a suitable solvent, such as hexane, and be allowed to pass through the Florisil ${ }^{T M}$ column. Separation of target analytes from interfering compounds is accomplished through elution time differences. Since PCBs are not retained on Florisil ${ }^{T M}$, the cleanup procedure simply involves collecting a suitable volume of the column effluent after the initial rinse portion is discarded to waste.

The sample cleanup process is integrated into the analytical platform through a fully automated column SLM. This SLM is built around the Gilson Syringe Pump Model 402 and Sampling Injector Model 231XL (Gilson Inc., Madison, WI 53562). The concentrated sample, from the HVC, is passed through the Florisil $^{\text {TM }}$ column, and the eluted sample is collected and transferred to a septum-capped vial.

EPA Method 8082 defines the parameters used to identify and calculate levels of concentration of various organochlorine pesticides and PCBs as Aroclors. The method is specific to analysis by capillary column gas chromatography. A known volume of sample is removed from the septum-capped vial and is injected into a GC. Target analytes are detected with an ECD. Based on retention times, detection of the 
target analytes produces an elution pattern that allows identification of the specific Aroclor as well as calculation of its concentration.

The GC SLM is a Varian Model 3400 CX (Walnut Creek, California) unit equipped with an ECD and a Model 8000 liquid autosampler. Control of the GC by the TSC is accomplished with an external process that interfaces to the Varian STAR software. The GC is equipped with a J\&W DB-5 HT ( $30 \mathrm{~m} \times 0.25 \mathrm{~mm}$ i.d. $\times 0.25 \mu \mathrm{m}$ liquid film) capillary column. The Perkin-Elmer/Nelson data acquisition system and the TurboChrom Version 4.0 data processing software run in parallel with the Varian GC; the TurboChrom system was not a SLM.

The system was calibrated with Aroclors 1242,1254 , and 1260 at the following concentrations: $0.05,0.10,0.20,0.40$, and $0.8 \mu \mathrm{g} / \mathrm{mL}$. All injections used $2 \mu \mathrm{L}$. Five signature peaks from each Aroclor were chosen for calibration and are included in the group reports. All peaks included in the calibration set had an $R^{2}$ value greater than 0.995 . Single chromatograms of Aroclors 1016, 1221, 1232, and 1248 were recorded in the event that a sample containing one of these Aroclors was encountered.

Daily calibration check standards used $0.40 \mu \mathrm{g} / \mathrm{mL}$ Aroclor standards.

The calibration for decachlorbiphenyl (DCBP) used the following concentrations: $0.02,0.04,0.08$, 0.16 , and $0.32 \mu \mathrm{g} / \mathrm{mL}$. All samples were spiked with DCBP using a $0.16 \mu \mathrm{g} / \mathrm{mL}$ standard.

The PCB standards were procured from ULTRA Scientific (North Kingstown, Rhode Island.

Samples are transported among the SLMs with the ORCA robot (Saigan, Inc., Indianapolis, Indiana) moving along a 3-m linear track.

\subsection{DATA MANAGEMENT PROCEDURES}

Each day's work was initiated by the preparation of a work sheet, which essentially was a sequence list. This information was entered into the SAM HCI and into the TurboChrom software system. Processing of the samples was performed in accordance with the information contained on the work sheet. Work was arranged such that daily calibration standards were ready for review by the analyst at the start of the work shift.

Hardcopy data packages where assembled according to the sample chain of custody sheets. Summaries of the results were forwarded to the firm performing the soil sampling at the David Witherspoon 1630 Site.

Data files for the DIM were produced by the Varian STAR GC system. The DIM was operated on-line using the data from the Varian STAR system. Because the performance of the DIM had not been validated, information generated by the DIM was used by the operational staff as a guide to assess system performance.

\subsubsection{TurboChrom}

All parameters associated with the acquisition of raw data, the building of calibration models, and data reporting were performed with the TurboChrom system. Practices followed during this project were similar to those used in the environmental analysis laboratory at LANL 


\subsubsection{Data Interpretation Module}

The fully automated SAM requires automation of the analysis of data produced by the AIM. For the PCB SAM, this task is accomplished with a fuzzy logic-based DIM. The DIM operates in two modes. One mode is an on-line mode that performs data analysis using the same set of state variables and protocols used by the hardware SLMs. The other mode is an off-line mode that provides the chemist with the tools required to build automated data processing methods, build calibration models, perform data assessment, and manually reanalyze sample data that the on-line mode was not able to analyze. The DIM also validates that the data received from the AIM is analyzable and performs a quality control evaluation on each chromatogram. The specific set of tasks performed by the DIM on a sample depends on the sample class: unknown, blank, control, or standard. These tasks are specified in the method that is attached to the sample when the sample in entered into the SAM through the HCI.

Operation of the DIM proceeds as follows. The SAM master controller initiates a sample by reading a method file containing the commands that define the total analysis protocol. When the AIM has completed the data acquisition and transferred the raw data to the source data directory for the DIM, the SAM master controller passes to the DIM the information necessary for the it to analyze the data. The transfer of data from the GC to the DIM occurs via the netCDF data exchange format, ${ }^{3}$ which is the basis of the chromatography data exchange standard adopted by the Analytical Instrument Association (AIA). ${ }^{4}$ The AIA standard makes the processing of data by the DIM independent of the GC vendor. However, the peak area data contained within the AIA formatted data file depend on the algorithms implemented by the vendor in the GC software and the specific values of settable parameters selected by the user. The results of the DIM are written into a space-delimited text file. A portion of the result information is returned to the master controller, which transfers it to the $\mathrm{HCI}$ for display.

The PCB DIM uses single and multivariate data processing algorithms to process each chromatogram. The DIM as implemented for this testing program generated an estimate of the analyte concentrations using five different data processing algorithms: (1) principal component regression using the raw chromatogram (PCRR), (2) principal component regression using peak area data (PCRP), (3) multiple linear regression using peak area data (MLRP), (4) simultaneous linear equations (SLEs) (i.e., singular value decomposition), and (5) a concentration artificial neural network (ANN). ${ }^{5,6}$ The MLRP algorithm is essentially identical to the data analysis algorithms embedded in all the commercial gas chromatography software. Studies with Aroclor mixtures have shown that although the peak-based algorithms are less susceptible to interferences, the full chromatogram algorithms are more accurate under varying baseline and Aroclor mixture situations. ${ }^{7}$ Except for the SLE algorithm, each data processing algorithm returns a value for the analyte concentration along with the $95 \%$ probability level confidence interval (CI).

3 "netCDF Users's Guide, An Interface for Data Access," Version 2.0, Unidata Program Center, Boulder, Colorado, October 1991.

4 “Analytical Data Interchange-Chromatography Data: Specification," Version 1.0, Analytical Instrument Association, Alexandria, Virginia 22314, 1992.

${ }_{5}^{5}$ Application of Artificial Neural Networks in Quantitative Analysis of Gas Chromatograms, M. A.

Williams, M.S. Thesis, Department of Electrical Engineering, University of Tennessee, Knoxville, December 1995.

6 "CANNS: The Concentration Artificial Neural Network System," M. A. Hunt, M. A. Williams, T. L. Lewis, and M. A. Abidi, Department of Electrical Engineering, University of Tennessee, Knoxville, January 1996.

7 "Automated Data Interpretation Applied to Multi-Compound Analytes," M. A. Hunt and L. N. Klatt, The Pittsburgh Conference, Atlanta, Georgia, March 1997. 
Each of the data processing algorithms also generates an "importance" parameter, which is the computed norm distance between the reconstructed data and the raw data. The importance parameter attempts to capture in a single scalar quantity how well a particular chromatogram is described by the calibration standards. The value of the importance parameter can range from 1.0 to negative infinity. Perfect description of an unknown chromatogram by the calibration standards yields an importance value of 1.0. As the degree of agreement between the unknown chromatogram and the calibration standards decreases, the value of the importance parameter decreases toward negative infinity. Typically, an importance value less than zero indicates that for that particular data process algorithm the unknown chromatogram is not well described by the calibration data set.

A fuzzy logic system is used to perform the result combination to produce the final analyte result. Fuzzy logic was selected to perform the result combination because it allows one to represent inexact, incomplete, and uncertain information in a mathematical framework. Fuzzy logic generalizes the binary set membership to the real interval $[0,1]$, and set membership can be represented in varying degrees (e.g., "Friday is a member of the set weekend at the level 0.5 "). Fuzzy logic systems can incorporate expert knowledge and are tolerant of imprecise data. These features of fuzzy logic fit well with the DIM because a precise relationship between the data processing algorithms and the unknown scenarios does not exist.

The fuzzy inference system receives as input the information generated by each data processing algorithm (i.e., analyte concentration, confidence interval, and importance parameter). This information is processed through the fuzzy membership functions and a set of combination rules to yield a weight factor. Figure 4.1 shows the operation of the fuzzy logic system.

The DIM attempts to encapsulate the known performance characteristics of each analytic method using the fuzzy memberships and rules defined in each fuzzy inference system. As shown in Fig. 4.1, each analytical method has a dedicated fuzzy inference system that generates a weighting factor for the combination of all the methods' results. The inference system works in the following manner. The input variables are defined, and membership functions are created for each variable. Membership functions map the value of the input variable to degrees of membership in a set of defined categories (e.g., for the input variable concentration, the sets are absent and present). Since the exact concentration at which an analyte is present is difficult to determine or is based on a regulatory limit, the transition between absent and present can be made gradual with the membership functions. The rules of the system are in the form of IF-THEN statements that determine the relationships between the input fuzzy sets and the output fuzzy sets. An example for the MLRP algorithm is "if analyte 1 is present and analyte 3 is present and importance is medium, then the weight factor is low." This rule captures the expert's knowledge that this method does not perform well under this scenario. The final step in the individual inference system is the defuzzification of the output. Each rule generates a resulting degree of membership in one of the output sets, and these sets are combined using a center-of-mass approach to yield a specific weight value. The weight values from each method's fuzzy inference system are normalized and used to combine the analyte's concentration and confidence interval. 


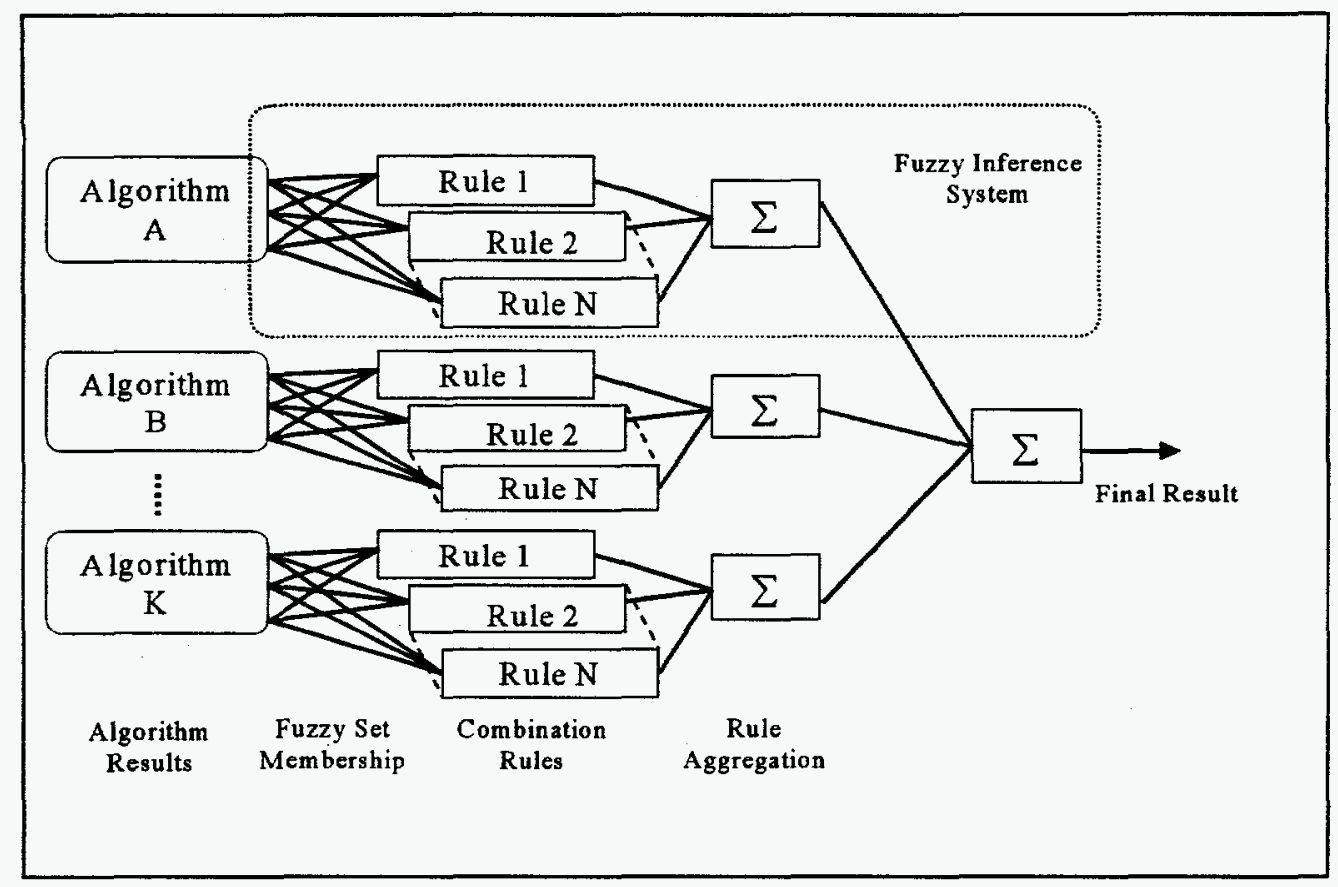

Fig. 4.1. Block diagram of DIM fuzzy logic operations.

The output of the fuzzy inference system is a weight factor applicable to the results from each of the data processing algorithms. The final analyte values are the weighted average of all the data processing algorithm values. The final confidence intervals are the weighted root-mean square of the confidence intervals from each of the data processing algorithms. Table 4.1 summarizes the information obtained from the fuzzy logic system for a typical sample. The information in the final row of the table is the information returned by the DIM to the SAM master controller, which in turn sends it to the HCI for display. In this particular sample, one concludes that Aroclor 1242 and 1260 are present but well below the regulatory level of concern. Because the confidence interval for Aroclor 1254 is significantly larger than the estimated concentration the presence of Aroclor 1254 in unlikely. The fuzzy logic summary table is saved in a file in the subdirectory containing the raw chromatogram file.

The DIM performs three quality assurance checks on each chromatogram: a signal off-scale check, retention time marker evaluation, and surrogate recovery. The signal off-scale check merely verifies that the raw detector signal is within the dynamic range of the analog-to-digital converter. The retention time marker evaluation and the surrogate recovery checks verify that the observed values are within the specified statistical control limits. The basis for establishing the statistical control limits are given in the EPA SW-846 Solid Waste Manual. If any one of these checks fails, the process of analyzing the chromatograms for the constituents of interest occurs only if the operator manually instructs the TSC to proceed with the analysis step. The results of each quality assurance check are returned by the DIM to the TSC, which sends it to the HCI for display. The results of the quality assurance checks are also saved in a file in the subdirectory containing the raw chromatogram file. 
Table 4.1. Fuzzy logic summary table

Used 425460 as the calibration data set.

Analyzed for the following analytes:

Aroclor 1242

Aroclor 1254

Aroclor 1260

Combination Mode: Fusion

Combination Matrix

\begin{tabular}{|l|c|c|c|c|c|c|c|c|}
\hline $\begin{array}{c}\text { Data- } \\
\text { Processing } \\
\text { Algorithm }\end{array}$ & $\begin{array}{c}\text { Importance } \\
\text { Value }\end{array}$ & $\begin{array}{c}\text { Fuzzy } \\
\text { Weight } \\
\text { Factor }\end{array}$ & $\begin{array}{c}\text { Aroclor } \\
1242 \\
(\mathrm{ppm})\end{array}$ & $\begin{array}{c}\text { Aroclor } \\
1242 \mathrm{CI}^{\mathrm{a}} \\
(\mathrm{ppm})\end{array}$ & $\begin{array}{c}\text { Aroclor } \\
1254 \\
(\mathrm{ppm})\end{array}$ & $\begin{array}{c}\text { Aroclor } \\
1254 \mathrm{CI} \\
(\mathrm{ppm})\end{array}$ & $\begin{array}{c}\text { Aroclor } \\
1260 \\
(\mathrm{ppm})\end{array}$ & $\begin{array}{c}\text { Aroclor } \\
1260 \mathrm{CI} \\
(\mathrm{ppm})\end{array}$ \\
\hline PCRR & -3.418 & 0.00002 & 0.501 & 0.110 & 0.235 & 0.059 & 0.414 & 0.023 \\
\hline PCRP & 0.746 & 0.687 & 0.104 & 0.034 & 0.010 & 0.030 & 0.104 & 0.029 \\
\hline MLRP & 0.724 & 0.657 & 0.074 & 0.047 & N.A $^{\mathrm{b}}$ & N.A. & N.A. & N.A. \\
\hline MLPR & 0.399 & 0.500 & N.A. & N.A. & 0.072 & 0.027 & N.A. & N.A. \\
\hline MLRP & 0.874 & 0.886 & N.A. & N.A. & N.A. & N.A. & 0.090 & 0.024 \\
\hline ANN & 0.856 & 0.665 & 0.071 & 0.037 & 0.000 & 0.078 & 0.092 & 0.036 \\
\hline SLE & 0.461 & 0.358 & 0.075 & N.A. & 0.013 & N.A. & 0.073 & N.A. \\
\hline $\begin{array}{l}\text { Final } \\
\text { Result }\end{array}$ & & & 0.082 & 0.039 & 0.021 & 0.052 & 0.092 & 0.029 \\
\hline
\end{tabular}

${ }^{a} \mathrm{CI}$ denotes the $95 \%$ probability confidence interval.

${ }^{b} \mathrm{~N}$.A. denotes that the value is not available from this data processing algorithm. 


\section{SYSTEM PERFORMANCE}

\subsection{CHEMICAL PERFORMANCE}

Data collected during analysis of the Witherspoon soil samples were used to evaluate the chemical performance of the PCB SAM system. The quality control (QC) measures used were based on those specified under EPAs SW-846, Chapter One, Sect. 4.4, "Laboratory QA and QC Procedures," as well as on specific criteria listed under Methods 3541, 8000, and 8082 for the analysis of PCBs in a soil matrix. The data acquired from QC procedures are used to estimate the quality of analytical data, to determine the need for corrective action in response to identified deficiencies, and to interpret results after corrective action procedures are implemented.

In addition, the data collected are used to demonstrate the degree to which the PCB SAM system is capable of meeting or exceeding the established chemical performance criteria developed for the manual implementation of the EPA methods used. Each of the following sections is a measure of how well the PCB SAM system performed during the Witherspoon project. Each section provides a summary of the performance of the PCB SAM system relative to expected performance criteria established under EPA SW-846.

\subsubsection{Blind Control Samples}

A blind control sample is a QC sample introduced into a process to monitor the performance of the system. The sample is fortified with the analyte being measured at a detectable concentration level in a matrix the same as or similar to the matrix of the samples being analyzed. A blind control sample is typically obtained from a third-party source. The analyte identity and concentration are unknown to the analyst. For the Witherspoon project, a batch of blind control samples were prepared by the CST-3 Quality Assurance Group at LANL. The CAA analyst routinely inserted one of these blind samples into the sample preparation stream along with the Witherspoon samples being processed for analysis. The results are summarized in Table 5.1.

The percent recovery values are well within the normal analytical operating parameters for this method. Typical acceptance criteria fall between 50 and $150 \%$. The average from the PCB SAM system performance was $100 \pm 32 \%$.

\subsubsection{Method Blanks}

A method blank is an analyte-free matrix to which all reagents are added in the same volumes or proportions as used in sample processing. The method blank is carried through the complete sample preparation and analytical procedure. The method blank is used to document contamination resulting from the analytical process. For a method blank to be acceptable for use with the accompanying samples, the concentration in the blank of any analyte of concern should not be higher than the highest of either (1) the method detection limit, (2) 5\% of the regulatory limit for that analyte, or (3) 5\% of the measured concentration in the sample. A method blank is analyzed with each batch of samples processed to assess contamination levels in the laboratory. For the Witherspoon project, a method blank was processed with each daily batch of samples. The method blank consisted of $10 \mathrm{~g} \mathrm{of} \mathrm{Na}_{2} \mathrm{SO}_{4}$ processed in the exact same manner as the soil samples. The results are listed in Table 5.2. 
Table 5.1. System performance on blind QC samples

\begin{tabular}{|c|c|c|c|c|c|}
\hline $\begin{array}{c}\text { QC } \\
\text { Sample ID }\end{array}$ & $\begin{array}{c}\text { Amount } \\
(\mathrm{ppm})\end{array}$ & $\begin{array}{c}\text { Recovery } \\
\%\end{array}$ & $\begin{array}{c}\text { QC } \\
\text { Sample ID }\end{array}$ & $\begin{array}{c}\text { Amount } \\
(\mathrm{ppm})\end{array}$ & $\begin{array}{c}\text { Recovery } \\
\%\end{array}$ \\
\hline 384 & 0.09 & 101 & 637 & 0.55 & 144 \\
\hline 387 & 0.36 & 88 & 639 & 0.18 & 160 \\
\hline 390 & 0.67 & 72 & 641 & 0.68 & 139 \\
\hline 628 & 0.05 & 64 & 645 & 0.30 & 143 \\
\hline 631 & 0.43 & 141 & 382 & 0.79 & 71 \\
\hline 635 & 0.18 & 100 & 385 & 0.35 & 80 \\
\hline 640 & 0.67 & 83 & 388 & 0.59 & 77 \\
\hline 642 & 0.81 & 79 & 391 & 0.15 & 78 \\
\hline 644 & 0.31 & 162 & 632 & 0.35 & 66 \\
\hline 647 & 0.56 & 93 & 634 & 0.64 & 63 \\
\hline 386 & 0.50 & 107 & 636 & 0.79 & 62 \\
\hline 389 & 0.20 & 110 & 638 & 0.20 & 81 \\
\hline 629 & 0.43 & 141 & 643 & 0.49 & 60 \\
\hline 630 & 0.80 & 141 & 646 & 0.05 & 112 \\
\hline 633 & 0.05 & 96 & & Mean $=$ & 100.5 \\
\hline & & & & Std. Dev. $=$ & 32.3 \\
\hline & & & & Minimum $=$ & 60 \\
\hline & & & & & 160 \\
\hline
\end{tabular}

Table 5.2. System performance on method blanks

\begin{tabular}{|c|c|c|c|}
\hline Date & $\begin{array}{c}\text { Total PCBs } \\
\text { Detected }(\mathrm{ppm})\end{array}$ & Date & $\begin{array}{c}\text { Total PCBs } \\
\text { Detected }(\mathrm{ppm})\end{array}$ \\
\hline $24-$ Sep & None & $24-$ Oct & None \\
\hline $25-$ Sep & None & $29-$-ct & None \\
\hline $7-$ Oct & None & $30-$ Oct & None \\
\hline 8 -Oct & None & $3-$ Nov & 0.55 \\
\hline $9-$ Oct & 0.09 & $4-$ Nov & 0.10 \\
\hline $10-$ Oct & None & $5-$ Nov & None \\
\hline $13-$ Oct & 0.06 & $6-$ Nov & None \\
\hline $14-$ Oct & None & $10-$ Nov & None \\
\hline $15-$ Oct & None & $11-$ Nov & None \\
\hline $16-$ Oct & None & $12-$ Nov & None \\
\hline $17-$ Oct & None & $13-$ Nov & None \\
\hline $20-$ Oct & None & $14-$ Nov & None \\
\hline $21-$ Oct & None & $19-$ Nov & None \\
\hline
\end{tabular}


As a general rule, the PCB SAM system was free of contamination, as indicated by the data in Table 5.2. The only data point that was not compliant with the EPA acceptance criteria was the blank on November 3. This was because of carryover from a previously analyzed soil sample that contained a total Aroclor content of $64 \mathrm{ppm}$. EPA protocol requires reanalysis of samples analyzed in the batch containing that blank. It is interesting to note that blind quality control sample 638 was run after the November 3 blank and its recovery was not elevated. This result suggests that the level of sample carryover in the system is less than $1 \%$. The concentrator SLM is the likely source of the sample carryover and the user can minimize analyte carryover by adjusting the SLM self-cleaning parameters.

\subsubsection{Daily Calibration Check Standards}

Calibration is a reproducible reference point to which all sample measurements can be correlated. A sound calibration program includes provisions for documenting the frequency, conditions, standards, and records reflecting the calibration history of a measurement system. The accuracy of the calibration is important because all data will be in reference to the standard calibration. The working calibration curve is verified on each working day by the injection of one or more calibration standards. The frequency of verification is a minimum of once every 20 samples or once every 12-hour operational shift. The EPA recommends that calibration verification standard results not exceed a difference of more than $\pm 15 \%$ difference when compared with the initial calibration curve. Individual laboratories have the option to set internal QC acceptance criteria based on the data quality objectives established by their customers. The CAA program set its acceptance criteria for this test to $\pm 20 \%$. A summary of the daily calibration checks is listed in Table 5.3. Plots of the individual Aroclor recovery for the daily calibrations, expressed as a difference from the ideal value of $100 \%$, are shown in Fig. 5.1.

Table 5.3. System performance on daily calibration standards

\begin{tabular}{|c|c|c|c|c|c|c|c|}
\hline \multicolumn{5}{|c|}{ Aroclor } & \multicolumn{3}{|c|}{ Aroclor } \\
\hline Date & 1242 & 1254 & 1260 & Date & 1242 & 1254 & 1260 \\
\hline 24-Sep & 105 & 102 & 102 & 27-Oct & 94 & 97 & 93 \\
\hline 25-Sep & 92 & 111 & 100 & 28-Oct & 99 & 103 & 94 \\
\hline 25-Sep & 111 & 107 & 96 & 29-Oct & 98 & 110 & 98 \\
\hline 7-Oct & 114 & 110 & 106 & 30-Oct & 107 & 106 & 94 \\
\hline 7-Oct & 121 & 111 & 111 & 31-Oct & 89 & 98 & 90 \\
\hline 8-Oct & 119 & 113 & 107 & 31-Oct & 98 & 97 & 87 \\
\hline 8-Oct & 111 & 114 & 99 & 4-Nov & 94 & 96 & 97 \\
\hline 9-Oct & 118 & 106 & 98 & 4-Nov & 94 & 105 & 103 \\
\hline 9-Oct & 115 & 109 & 95 & 6-Nov & 92 & 101 & 94 \\
\hline 14-Oct & 109 & 108 & 104 & 6-Nov & 94 & 99 & 89 \\
\hline 15-Oct & 112 & 116 & 111 & 7-Nov & 88 & 98 & 91 \\
\hline 16-Oct & 107 & 115 & 110 & 11-Nov & 115 & 118 & 118 \\
\hline $20-\mathrm{Oct}$ & 82 & 85 & 89 & 13-Nov & 97 & 104 & 98 \\
\hline $20-$ Oct & 89 & 93 & 86 & 13-Nov & 85 & 109 & 107 \\
\hline 21-Oct & 86 & 96 & 89 & 14-Nov & 98 & 108 & 106 \\
\hline 21-Oct & 84 & 95 & 89 & 18-Nov & 90 & 114 & 94 \\
\hline $22-O c t$ & 86 & 96 & 88 & 19-Nov & 88 & 105 & 101 \\
\hline 23-Oct & 89 & 95 & 87 & & & & \\
\hline 24-Oct & 102 & 103 & 97 & Mean $=$ & 99 & 104 & 98 \\
\hline 27-Oct & 100 & 103 & 94 & $\mathrm{~S}=$ & 11 & 8 & 8 \\
\hline
\end{tabular}




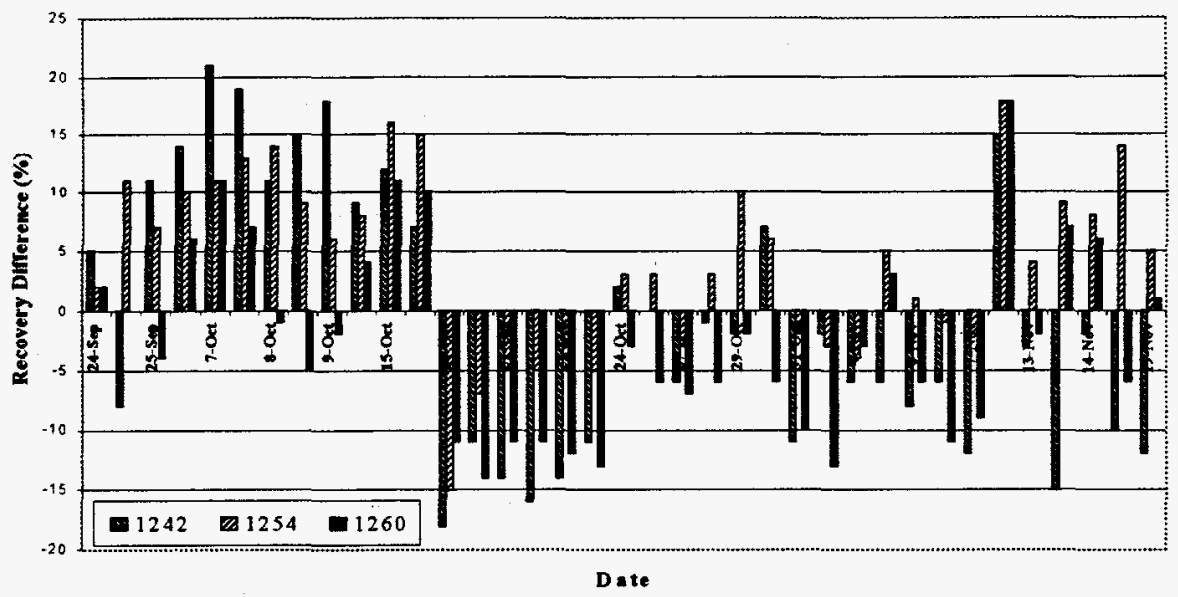

Fig. 5.1. System performance on daily calibration standards.

The daily calibration checks for the Witherspoon project met the quality assurance (QA) acceptance criteria established for this test by the CAA project team. The daily calibration check is strictly a QA test of the performance of the measurement system (i.e., the GC SLM). It is in no way indicative of the performance of the other modules in the PCB SAM.

\subsubsection{Matrix Spike and Matrix Spike Duplicate Samples}

The sample matrix, defined as the component or substrate that contains the analyte of interest (e.g., soil, water, or sludge etc.), can affect, or interfere with, the analytical measurement for possible analytes contained in that matrix. The deviation caused by the matrix effects of the measured value is known as matrix bias. It can be assessed by comparing a measured value to an accepted reference value in a sample of known concentration or by determining the recovery of a known amount of contaminant spiked into a sample matrix (matrix spike). In addition, the matrix spike and matrix spike duplicate results are used to determine the precision of the method for a specific matrix. Precision is the agreement among a set of replicate measurements without assumption of knowledge of the true value. For small sets of data, the relative percent difference (RPD) between two measurements is an estimate of the precision of the measurement process.

A matrix spike is an aliquot of a sample spiked with a known concentration of the target analyte(s). The spiking occurs prior to sample preparation and analysis. A matrix spike is used to document the bias of a method in a given sample matrix. A matrix spike duplicate is an intralaboratory split sample spiked with identical concentrations of the target analyte(s) used in the matrix spike. A matrix spike duplicate is used to document the precision and bias of a method in a given sample matrix. For the Witherspoon project, a matrix spike and matrix spike duplicate were prepared with every batch of 20 samples using Aroclor 1260 at a concentration of $0.40 \mathrm{ppm}$. The results are listed in Table 5.4.

The performance of the PCB SAM system in terms of precision and accuracy are well within, if not exceeding, the acceptance criteria for this QA test. Because of the high concentration of PCBs contained in sample 138, the matrix spike concentration level was insignificant relative to the total PCB concentration in the sample. Therefore, this data point was excluded from the summary statistics. 
Table 5.4. System performance on matrix spikes and spike duplicates

\begin{tabular}{|c|c|c|c|c|c|}
\hline $\begin{array}{l}\text { Sample } \\
\text { Number }\end{array}$ & $\begin{array}{c}\text { Recovery } \\
\%\end{array}$ & Bias & $\begin{array}{l}\text { Precision } \\
\text { RPD }\end{array}$ & $\begin{array}{c}\text { Average } \\
\text { Recovery \% }\end{array}$ & Std. Dev. \\
\hline M020 & 67.5 & -0.13 & & & \\
\hline D020 & 72.5 & -0.11 & 7.1 & 70 & 3.5 \\
\hline M051 & 85.0 & -0.06 & & & \\
\hline D051 & 112.5 & 0.05 & 31.4 & 99 & 19.4 \\
\hline M068 & 180.0 & 0.32 & & & \\
\hline D068 & 177.5 & 0.31 & 1.4 & 179 & 1.8 \\
\hline M109 & 90.0 & -0.04 & & & \\
\hline D109 & 100.0 & 0 & 10.5 & 95 & 7.1 \\
\hline M117 & 55.0 & -0.18 & & & \\
\hline D117 & 75.0 & -0.1 & 30.8 & 65 & 14.1 \\
\hline $\mathrm{M} 123$ & 105.0 & 0.02 & & & \\
\hline D123 & 120.0 & 0.08 & 6.7 & 113 & 10.6 \\
\hline M138 & 2300.0 & 8.8 & & & \\
\hline D138 & 2225.0 & 8.5 & 3.3 & $2263^{a}$ & 53.0 \\
\hline M161 & 152.5 & 0.21 & & & \\
\hline D161 & 112.5 & 0.05 & 30.2 & 133 & 28.3 \\
\hline M200 & 85.0 & -0.06 & & & \\
\hline D200 & 87.5 & -0.05 & 2.9 & 86 & 1.8 \\
\hline M211 & 142.5 & 0.17 & & & \\
\hline D211 & 115.0 & 0.06 & 21.4 & 129 & 19.4 \\
\hline M227 & 117.5 & 0.07 & & & \\
\hline D227 & 140.0 & 0.16 & 17.5 & 129 & 15.9 \\
\hline Mean $=$ & 109.6 & $0.04^{\mathrm{a}}$ & 14.8 & $110^{\mathrm{a}}$ & $12^{\mathrm{a}}$ \\
\hline Std. Dev. $=$ & $33.9^{\mathrm{a}}$ & & & & \\
\hline
\end{tabular}

${ }^{a}$ Sample 138 was excluded from the statistical parameter.

\subsubsection{Surrogate Recovery}

A surrogate is an organic compound that is similar to the target analyte(s) in chemical composition and behavior in the analytical process but that is not normally found in environmental samples. The surrogate compound used for the Witherspoon project was DCBP. The surrogate is added to each sample aliquot prior to extraction and is used as a real-time check on the performance of the method for the analyte(s) of interest. The percent recoveries of the surrogate by sample type are listed in Table 5.5 .

The performance of the PCB SAM system in terms of surrogate recoveries is well within the acceptance criteria for this QA test. These data would typically be used to establish a baseline of performance for the system. The upper and lower control limits would be plus/minus two standard deviations about the mean percent recovery. 
Table 5.5. System performance on surrogate recovery

\begin{tabular}{|c|c|c|c|}
\hline Sample Type & Average & Std. Dev. & Count \\
\hline Unknowns & 63 & 17 & 234 \\
\hline Blanks & 80 & 12 & 25 \\
\hline $\begin{array}{c}\text { Matrix Spike-Matrix } \\
\text { Spike Duplicates }\end{array}$ & 70 & 14 & 22 \\
\hline Blind QCs & 85 & 7 & 29 \\
\hline Mean & 74 & 16 & \\
\hline
\end{tabular}

\subsubsection{Field Duplicate Samples}

These are independent samples that are collected as close as possible to the same point in space and time. They are two separate samples taken from the same source, stored in separate containers, and analyzed independently. These duplicates are useful in documenting the precision of the sampling process. Normally a laboratory is unaware of field duplicates at the time of analysis; however, the contractor performing the sample operation provided this information on the sample chain-of-custody sheets. For the Witherspoon project, a field duplicate was collected after every 20 th sample. The results of analysis for the field duplicates are listed in Table 5.6 for both the CAA PCB SAM system and the commercial laboratory. The entries with a zero value were samples that each laboratory reported as nondetects; these values were excluded from the statistical summary.

Table 5.6. System performance on field duplicates

\begin{tabular}{|c|c|c|}
\hline $\begin{array}{c}\text { Field Duplicate } \\
\text { Number }\end{array}$ & $\begin{array}{c}\text { Relative Difference } \\
\text { (\%) } \\
\text { CAA }\end{array}$ & $\begin{array}{c}\text { Relative Difference } \\
\text { (\%) } \\
\text { Lab }\end{array}$ \\
\hline 1 & 25 & 18 \\
\hline 20 & 0 & 0 \\
\hline 40 & 21 & 24 \\
\hline 60 & 26 & 85 \\
\hline 80 & 0 & 0 \\
\hline 100 & 0 & 0 \\
\hline 120 & 0 & 0 \\
\hline 140 & 1 & 29 \\
\hline 160 & 75 & 27 \\
\hline 180 & 55 & 103 \\
\hline Mean $=$ & 33.8 & 47.7 \\
\hline Std. Dev. $=$ & 26.6 & 36.5 \\
\hline
\end{tabular}

Interlaboratory comparisons based on field duplicate results are difficult to interpret. This is based on the uncertainies associated with the field sampling methodologies and the nonhomogeneous nature of the soil matrix. Based on the limited number of field duplicates that contained a measurable amount of PCBs, the CAA SAM performed as well as or exceeded the performance of the commercial laboratory. 


\subsubsection{Witherspoon Soil Samples}

A total of 234 soil samples received during the Witherspoon project were analyzed by the CAA analytical team. An assessment of the chemical performance of the CAA PCB SAM system compared with the manual processing of the same samples can be made using the data generated by the commercial laboratory for the Witherspoon project. Histograms of the PCB concentration in the samples analyzed are shown in Fig. 5.2 for both the commercial laboratory and the CAA PCB SAM system. Note that the distribution patterns for the CAA PCB SAM system and the commercial laboratory are essentially identical. This suggests that the two sets of results are in good agreement with each other. Approximately $70 \%$ of the samples contained less than $0.5 \mathrm{ppm}$ of PCBs. The correlation coefficient $\left(\mathrm{R}^{2}\right)$ between the two sets of results is 0.923 . A paired $t$-test was performed on the same set of data. This tests the hypothesis that the mean of the difference between the pairs of data for each sample analyzed is equal to zero (null=0). The results of the paired $t$-test are presented in Table 5.7. Based on the small mean difference and the large P-value, we can accept the hypothesis that there is no statistical difference between the CAA PCB SAM system and the commercial laboratory analytical results for the Witherspoon set of soil samples.

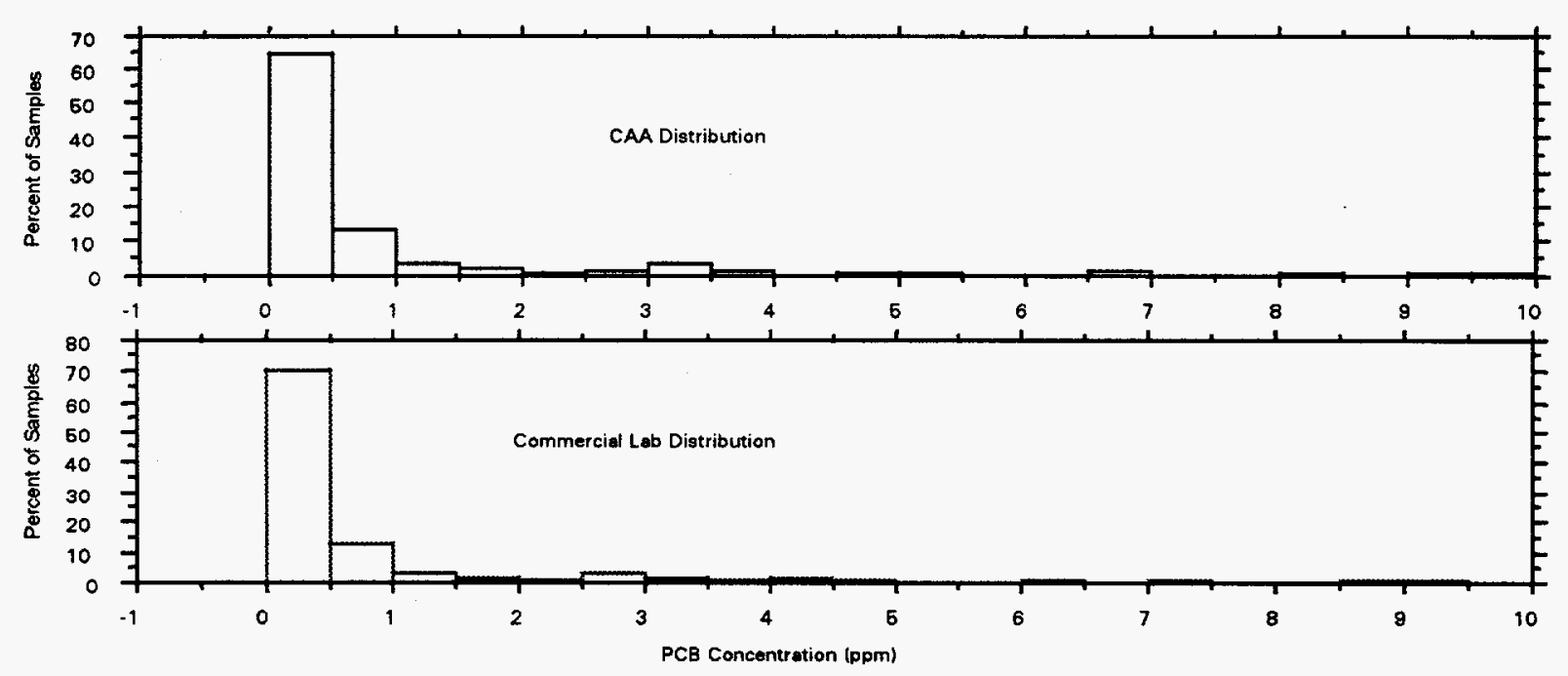

Fig. 5.2. Histogram distribution of PCBs in Witherspoon soil samples.

Table 5.7. Summary of paired $t$-test comparing CAA with the commercial laboratory

\begin{tabular}{|c|c|c|c|c|c|c|}
\hline Paired Data & $\begin{array}{c}\text { Mean } \\
\text { Difference }\end{array}$ & $\begin{array}{c}\text { Degrees of } \\
\text { Freedom }\end{array}$ & $\mathrm{t}$-Value & P-value & $95 \%$ Lower & $95 \%$ Upper \\
\hline CAA/Lab & -2.004 & 184 & -.480 & .6316 & -10.235 & 6.227 \\
\hline
\end{tabular}

To better quantify the performance of the CAA PCB SAM system, a calculation was performed to determine the percent difference between the concentration value of the CAA PCB SAMs and the average of all reported values (PCB SAM and the commercial laboratory). This histogram is shown in Fig. 5.3 and demonstrates that more than $50 \%$ of the samples have a $20 \%$ or less difference between the CAA PCB 
SAM value and the average value. Given the small concentration values, the nature of the soil matrix, and the analytical uncertainties associated with the method of analysis, this is an excellent indication that the CAA PCB SAM system performed equal to the manual method of analysis.

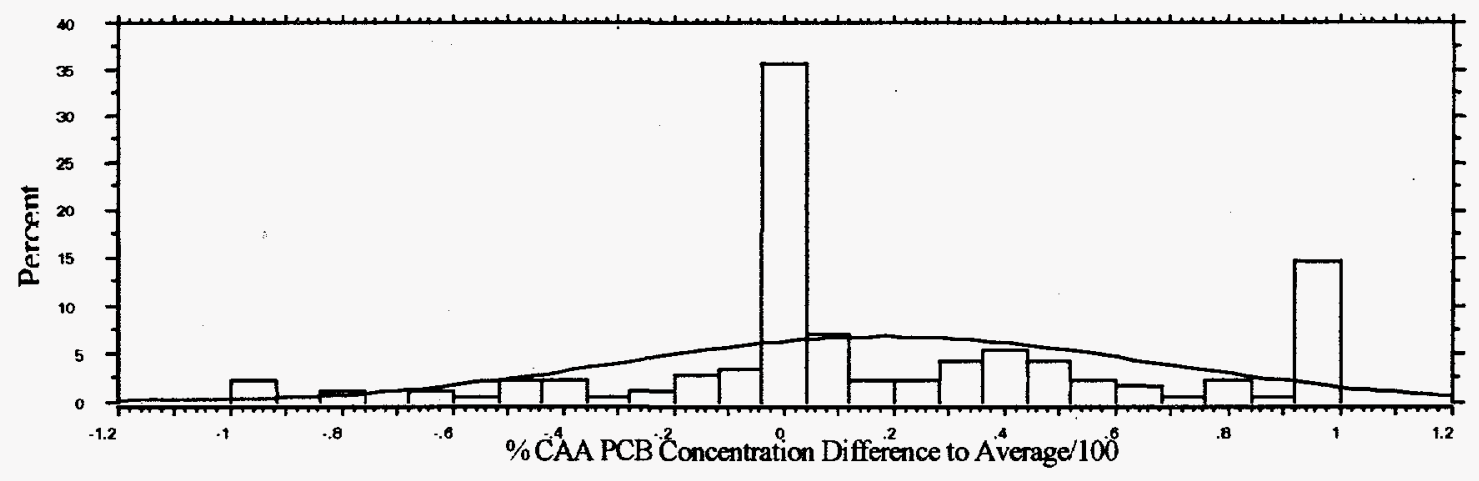

Figure 5.3: Histogram of CAA PCB Concentration Difference.

\subsubsection{Method Detection Limit and Quantitation Limit}

The QA practices specified by the EPA in the SW-846 method guidance document stipulate that method detection limit (MDL) information should be maintained for all methods performed in the laboratory. The purpose of such data is to demonstrate the proficiency of each analytical method routinely used in the laboratory.

The MDL for a method is defined as the minimum concentration of a substance that can be measured and reported with $99 \%$ confidence that the analyte concentration is greater than zero. This value is determined from analysis of a sample in a given matrix type containing the analyte. For operational purposes, calculating the MDL for a specific analytical method is a statistical exercise. It uses analyte recovery data obtained from the analysis of a series of matrix spikes at a concentration level three to five times the estimated MDL. In the Witherspoon project, seven replicate matrix spikes of $0.10 \mathrm{ppm}$ of Aroclor 1242 were used to calculate the MDL for the PCB SAM system. As such, the calculated MDL for the PCB SAM system was $0.022 \mathrm{ppm}$. EPA SW-846 Method 8082 proficiency guidelines stipulate achieving an MDL in the range of 0.057 to $0.070 \mathrm{ppm}$ for PCBs in a soil matrix. The PCB SAM system clearly exceeds the method proficiency requirements of EPA SW-846 Method 8082.

In contrast to the MDL, the estimated quantitation limit (EQL) for a method is the lowest concentration that can be reliably achieved within the specified limits of precision and accuracy during routine laboratory operating conditions. The EQL is generally 5 to 10 times the MDL. For many analytes the EQL is selected as the lowest nonzero standard in the calibration curve. Sample EQLs are highly matrix dependent. Given this, on the basis of a dry, 10-gram sample, the PCB SAM system's EQL is the lowest point on the calibration curve $(0.05 \mathrm{ppm})$ for each Aroclor used during the Witherspoon project.

\subsubsection{PCB Data Interpretation Module Performance}

Previous work to evaluate the DIM involved working with standard solutions of known Aroclor content. No work has been done to validate the performance of the DIM with a large set of environmental 
samples. Development testing of the CAA PCB SAM using samples obtained from the David Witherspoon 1630 Site has provided the opportunity to conduct the validation. This is an ongoing activity, and the full results will be published in a separate report. The material included in this report provides preliminary performance data on three sample types: daily calibration check standards, blind QC samples, and a limited subset of the soil samples.

The DIM data reported herein are the result of postprocessing the TurboChrom-derived data files through the DIM under the control of the TSC. This approach allows one to make a direct comparison between the two software systems without concerns for differences in the source of the raw data that may be related to differences in peak integration algorithms implemented by different vendors. During developmental testing of the CAA PCB SAM, the DIM used the AIA-formatted data files obtained from the Varian GC. Early in the testing it became apparent that the results being reported by the DIM were in disagreement with those being reported by the TurboChrom system. The DIM development team thinks this difference is related to the use of a less than optimum set of data acquisition and processing parameters on the Varian GC. A thorough reevaluation of the Varian GC derived data is planned.

\subsubsection{Daily calibration standards}

A total of 37 sets of calibration check standards were processed on 26 separate operating days. The average recoveries calculated by the DIM and TurboChrom software systems for these samples were essentially identical (e.g., the DIM- and TurboChrom-derived results for Aroclor 1254 were $100 \pm 7$ and $104 \pm 8 \%$, respectively). Of the 111 standards 5 would have been flagged as failing the 80 to $120 \%$ recovery acceptance criterion but by only 1 to $3 \%$ percentage points. During the actual processing of the sample, TurboChrom flagged one Aroclor 1242 check standard as not meeting the acceptance criterion, but by only 1 percentage point. The failure detected by TurboChrom was one of those flagged by the DIM. In all but one instance only one Aroclor check standard failed the acceptance criterion. Because the surrogate recoveries were within the normal range and the other Aroclor check standards met the acceptance criterion, the GC calibration was judged as valid. The fact that the next calibration check standard met the acceptance criterion verified the correctness of the earlier decision. The recovery data are plotted in Fig. 5.4. Comparison of the TurboChrom results shown in Fig. 5.1 with the DIM results shows that there is essentially no difference in the performance of the two data processing software systems.

\subsubsection{Blind quality control standards}

A total of 29 blind QC standards were processed during the development test. Tables 5.8 through 5.10 compare the results obtained from the QC sample set for Aroclors 1242, 1254, and 1260, respectively, for the TurboChrom and DIM software systems. The $\mathrm{CI}$ values are for the $95 \%$ probability level. The CIs for the TurboChrom results are based on the replicate values contained in the group report for each Aroclor; the CIs for the DIM are the mean-squared weighted values derived from the multiple data processing algorithms, as illustrated in Table 4.1.

The DIM software reported slightly lower surrogate recovery. This small difference is because the chemist used only the 5 DCBP peaks obtained from the Aroclor 1242 standards to build the surrogate calibration model for TurboChrom, while the DIM used all 15 calibration chromatograms. 


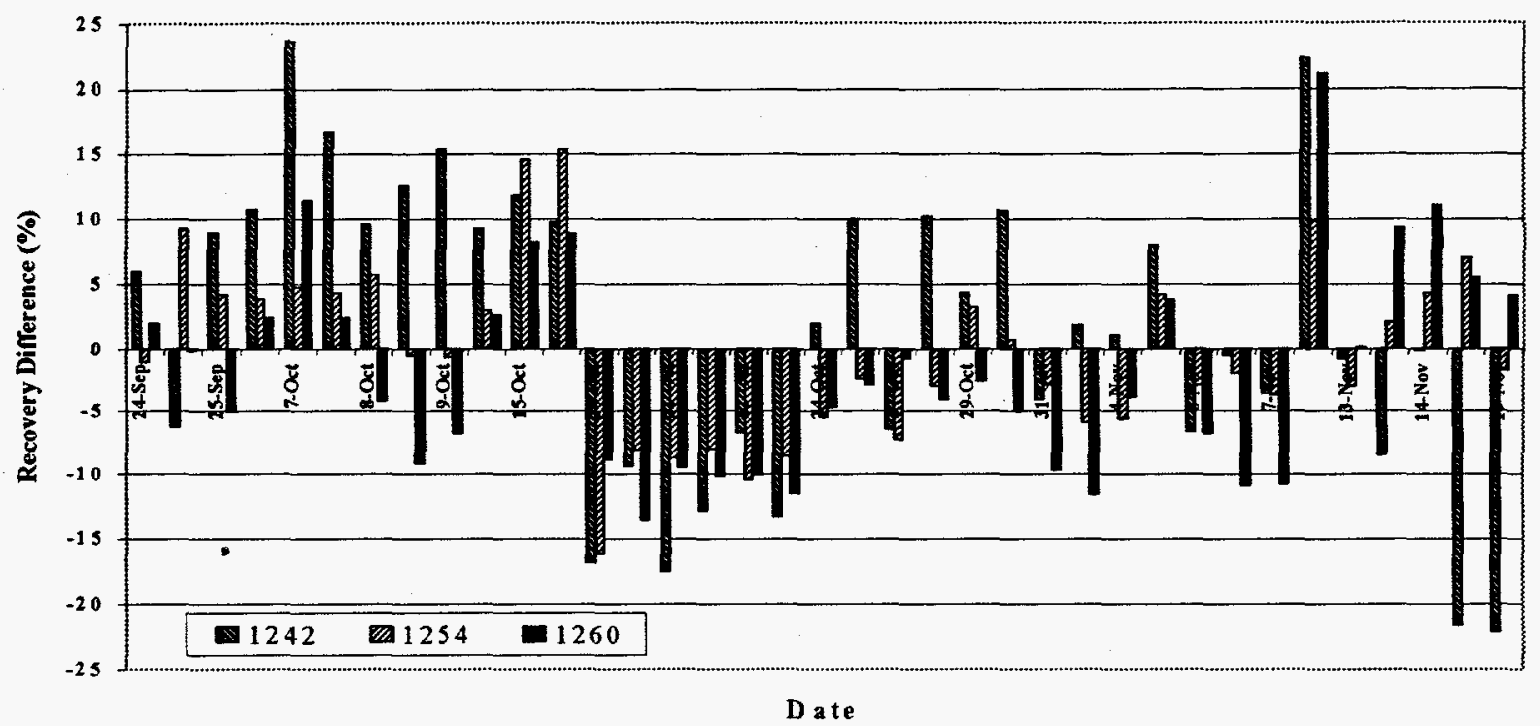

Fig. 5.4. DIM performance on daily calibration standards.

Based on the application of the F-test, the variances of the errors for the Aroclor values derived from the TurboChrom and the DIM software systems are not statistically different. Application of the $t$ test to the means errors shows that the Aroclor values derived from the TurboChrom and DIM software systems are not statistically different.

Table 5.8. Aroclor 1242 in blind QC Samples

\begin{tabular}{|c|c|c|c|c|c|c|c|c|c|c|}
\hline $\begin{array}{c}\text { QC } \\
\text { Sample }\end{array}$ & $\begin{array}{c}\text { Aroclor } \\
1242 \\
(\mathrm{ppm})\end{array}$ & $\begin{array}{c}\text { TC } \\
\text { Result } \\
(\mathrm{ppm})\end{array}$ & $\begin{array}{c}\text { TC } \\
\mathrm{CI} \\
(\mathrm{ppm})\end{array}$ & $\begin{array}{c}\text { DIM } \\
\text { Result } \\
(\mathrm{ppm})\end{array}$ & $\begin{array}{c}\text { DIM } \\
\mathrm{CI} \\
(\mathrm{ppm})\end{array}$ & $\begin{array}{c}\text { TC } \\
\text { Sur. Rec. } \\
(\%)\end{array}$ & $\begin{array}{c}\text { DIM } \\
\text { Sur. Rec. } \\
(\%)\end{array}$ & $\begin{array}{c}\text { TC } \\
\text { Error } \\
(\mathrm{ppm})\end{array}$ & $\begin{array}{c}\text { DIM } \\
\text { Error } \\
(\mathrm{ppm})\end{array}$ & $\begin{array}{c}\text { DIM -TC } \\
(\mathrm{ppm})\end{array}$ \\
\hline 384 & 0.09 & 0.09 & 0.16 & 0.05 & 0.03 & 74.6 & 72.9 & 0 & -0.04 & -0.05 \\
\hline 387 & 0.36 & 0.32 & 0.17 & 0.18 & 0.03 & 89.2 & 87.1 & -0.04 & -0.18 & -0.14 \\
\hline 390 & 0.67 & 0.48 & 0.05 & 0.49 & 0.04 & 89.1 & 87.1 & -0.19 & -0.19 & 0 \\
\hline 628 & 0.04 & 0.03 & 0.04 & 0.03 & 0.02 & 82.6 & 80.7 & -0.02 & -0.01 & 0 \\
\hline 631 & 0.43 & 0.60 & 0.16 & 0.66 & 0.04 & 83.1 & 81.2 & 0.17 & 0.23 & 0.06 \\
\hline 635 & 0.18 & 0.18 & 0.16 & 0.18 & 0.03 & 98.1 & 95.8 & 0 & 0 & 0 \\
\hline 640 & 0.67 & 0.56 & 0.26 & 0.59 & 0.04 & 92.2 & 90.1 & -0.11 & -0.09 & 0.02 \\
\hline 642 & 0.81 & 0.64 & 0.32 & 0.66 & 0.04 & 90.4 & 88.3 & -0.17 & -0.14 & 0.03 \\
\hline 644 & 0.30 & 0.50 & 0.15 & 0.52 & 0.04 & 86.7 & 84.7 & 0.19 & 0.21 & 0.02 \\
\hline 647 & 0.56 & 0.52 & 0.32 & 0.55 & 0.04 & 87.9 & 85.8 & -0.04 & -0.01 & 0.03 \\
\hline & & & & & Mean & 87.4 & 85.4 & -0.02 & -0.02 & 0 \\
\hline & & & & & Std. & 6.3 & 6.2 & 0.13 & 0.15 & 0.06 \\
\hline
\end{tabular}


However, a difference in performance between the TurboChrom and DIM software systems is noted when the patterns contained within the chromatograms are coupled with the criteria that a reportable result must have a value greater than the $\mathrm{CI}$. In this scenario, TurboChrom yielded two false negative results (QC samples 384 and 633) and one incorrect identification; the TurboChrom results for QC sample 628 yielded $0.03 \pm 0.01 \mathrm{ppm}$ of Aroclor 1260 . The DIM software did not yield any false negatives or any incorrect identifications.

Table 5.9. Aroclor 1254 in blind QC samples

\begin{tabular}{|c|c|c|c|c|c|c|c|c|c|c|}
\hline $\begin{array}{c}\text { QC } \\
\text { Sample }\end{array}$ & $\begin{array}{c}\text { Aroclor } \\
1254 \\
(\mathrm{ppm})\end{array}$ & $\begin{array}{c}\text { TC } \\
\text { Result } \\
(\mathrm{ppm})\end{array}$ & $\begin{array}{c}\text { TC } \\
\mathrm{CI} \\
(\mathrm{ppm})\end{array}$ & $\begin{array}{c}\text { DIM } \\
\text { Result } \\
(\mathrm{ppm})\end{array}$ & $\begin{array}{c}\text { DIM } \\
\mathrm{CI} \\
(\mathrm{ppm})\end{array}$ & $\begin{array}{c}\text { TC } \\
\text { Sur. Rec. } \\
(\%)\end{array}$ & $\begin{array}{c}\text { DIM } \\
\text { Sur. Rec. } \\
(\%)\end{array}$ & $\begin{array}{c}\text { TC } \\
\text { Error } \\
(\mathrm{ppm})\end{array}$ & $\begin{array}{c}\text { DIM } \\
\text { Error } \\
(\mathbf{p p m})\end{array}$ & $\begin{array}{c}\text { DIM -TC } \\
(\mathrm{ppm})\end{array}$ \\
\hline 386 & 0.50 & 0.54 & 0.26 & 0.54 & 0.06 & 76.2 & 74.5 & 0.04 & 0.04 & 0 \\
\hline 389 & 0.20 & 0.22 & 0.13 & 0.22 & 0.06 & 70.3 & 68.7 & 0.02 & 0.02 & 0 \\
\hline 629 & 0.43 & 0.60 & 0.07 & 0.60 & 0.04 & 89.1 & 87.1 & 0.18 & 0.17 & -0.01 \\
\hline 630 & 0.80 & 1.14 & 0.12 & 0.95 & 0.10 & 89.7 & 87.6 & 0.33 & 0.15 & -0.18 \\
\hline 633 & 0.05 & 0.05 & 0.06 & 0.05 & 0.05 & 83.4 & 81.5 & 0 & 0 & 0 \\
\hline 637 & 0.55 & 0.80 & 0.40 & 0.76 & 0.08 & 86.8 & 84.8 & 0.24 & 0.21 & -0.03 \\
\hline 639 & 0.18 & 0.28 & 0.18 & 0.26 & 0.03 & 85.7 & 83.7 & 0.10 & 0.09 & -0.02 \\
\hline 641 & 0.68 & 0.94 & 0.51 & 0.78 & 0.10 & 74.9 & 73.2 & 0.27 & 0.10 & -0.17 \\
\hline 645 & 0.30 & 0.43 & 0.23 & 0.43 & 0.03 & 76.7 & 74.9 & 0.13 & 0.13 & 0 \\
\hline & & & & & Mean & 81.4 & 79.6 & 0.15 & 0.10 & -0.05 \\
\hline & & & & & Std. & 7.0 & 6.8 & 0.12 & 0.07 & 0.07 \\
\hline
\end{tabular}

Table 5.10. Aroclor 1260 in blind QC samples

\begin{tabular}{|c|c|c|c|c|c|c|c|c|c|c|}
\hline $\begin{array}{c}\text { QC } \\
\text { Sample }\end{array}$ & $\begin{array}{c}\text { Aroclor } \\
1254 \\
(\mathrm{ppm})\end{array}$ & $\begin{array}{c}\text { TC } \\
\text { Result } \\
(\mathrm{ppm})\end{array}$ & $\begin{array}{c}\text { TC } \\
\text { CI } \\
(\mathrm{ppm})\end{array}$ & $\begin{array}{c}\text { DIM } \\
\text { Result } \\
(\mathrm{ppm})\end{array}$ & $\begin{array}{c}\text { DIM } \\
\text { CI } \\
(\mathrm{ppm})\end{array}$ & $\begin{array}{c}\text { TC } \\
\text { Sur. Rec. } \\
(\%)\end{array}$ & $\begin{array}{c}\text { DIM } \\
\text { Sur. Rec. } \\
(\%)\end{array}$ & $\begin{array}{c}\text { TC } \\
\text { Error } \\
(\mathrm{ppm})\end{array}$ & $\begin{array}{c}\text { DIM } \\
\text { Error } \\
(\mathrm{ppm})\end{array}$ & $\begin{array}{c}\text { DIM -TC } \\
(\mathrm{ppm})\end{array}$ \\
\hline 382 & 0.79 & 0.56 & 0.05 & 0.50 & 0.06 & 89.9 & 87.8 & -0.23 & -0.29 & -0.06 \\
\hline 385 & 0.35 & 0.28 & 0.03 & 0.28 & 0.03 & 96.5 & 94.4 & -0.07 & -0.07 & 0 \\
\hline 388 & 0.59 & 0.46 & 0.06 & 0.47 & 0.05 & 95.2 & 93.1 & -0.13 & -0.12 & 0.01 \\
\hline 391 & 0.15 & 0.12 & 0.01 & 0.12 & 0.03 & 92.2 & 89.9 & -0.03 & -0.03 & 0 \\
\hline 632 & 0.35 & 0.23 & 0.01 & 0.24 & 0.02 & 84.8 & 82.9 & -0.12 & -0.11 & 0.01 \\
\hline 634 & 0.64 & 0.40 & 0.05 & 0.49 & 0.05 & 86.0 & 84.0 & -0.24 & -0.15 & 0.08 \\
\hline 636 & 0.79 & 0.49 & 0.06 & 0.56 & 0.05 & 81.9 & 80.1 & -0.30 & -0.23 & 0.08 \\
\hline 638 & 0.20 & 0.16 & 0.02 & 0.16 & 0.05 & 69.6 & 67.9 & -0.04 & -0.04 & 0 \\
\hline 643 & 0.49 & 0.30 & 0.03 & 0.36 & 0.05 & 85.8 & 83.8 & -0.19 & -0.13 & 0.06 \\
\hline 646 & 0.05 & 0.06 & 0.01 & 0.06 & 0.03 & 82.4 & 80.4 & 0.01 & 0.01 & 0 \\
\hline & & & & & Mean & 86.4 & 84.4 & -0.14 & -0.12 & 0.02 \\
\hline & & & & & Std. & 7.8 & 7.6 & 0.10 & 0.09 & 0.04 \\
\hline
\end{tabular}




\subsubsection{Soil samples}

Thirteen soil sample chromatograms were randomly selected for postprocessing by the DIM. For the purposes of this report, the DIM will be evaluated using the following performance measures: surrogate recovery, identification of Aroclors, agreement between the results obtained with TurboChrom and the DIM for individual Aroclors, and the total Aroclor content in the samples as reported by TurboChrom and the DIM.

The date contained in Table 5.11 compares surrogate recovery results obtained from both the DIM and TurboChrom. The two software systems yielded identical results. Note that the very low surrogate recovery reported for Sample 10 was flagged by the DIM and the sample processing task was terminated.

Table 5.11. Surrogate recovery in soil samples as determined by the DIM and TC4 software systems

\begin{tabular}{|c|c|c|}
\hline Sample ID & $\begin{array}{c}\text { DIM } \\
\text { Surrogate } \\
\text { Recovery } \\
(\%)\end{array}$ & $\begin{array}{c}\text { TC4 } \\
\text { Surrogate } \\
\text { Recovery } \\
(\%)\end{array}$ \\
\hline 1 & 82 & 84 \\
\hline 2 & 83 & 85 \\
\hline 3 & 77 & 79 \\
\hline 4 & 71 & 73 \\
\hline 5 & 54 & 55 \\
\hline 6 & 56 & 57 \\
\hline 7 & 86 & 88 \\
\hline 8 & 35 & 36 \\
\hline 9 & 52 & 53 \\
\hline 10 & 7 & 7 \\
\hline 11 & 49 & 50 \\
\hline 12 & 26 & 26 \\
\hline 13 & 79 & 81 \\
\hline Mean $=$ & 62 & 64 \\
\hline Std. Dev. $=$ & 20 & 21 \\
\hline
\end{tabular}

In the on-line application of the DIM, the operator would be informed of the very low surrogate recovery and appropriate action would need to be taken before the data analysis was completed. During the postprocessing effort, the operator elected not to proceed with the data analysis. The very low surrogate recovery is the result of the analyst failing to add the surrogate spike to the extract when the original extract was diluted to bring the Aroclor concentrations within the limits of the calibration. In this situation the reported surrogate recovery data would be taken from the chromatogram obtained with the original extract. As with the blind QC samples, the DIM reported slightly lower surrogate recoveries (the reason for this is discussed in Sect. 5.1.9.2). 
Table 5.12 summarizes the results of the analyte identification exercise. The criteria used to determine that an Aroclor was present in the sample, indicated by a "Yes" in the table, included (1) the calculated concentration value must have been greater than zero, (2) the calculated concentration value must have been greater than the CI, and (3) the calculated concentration value must have been greater than the estimated quantitation limit for the system. Across all three Aroclors, the DIM and TurboChrom agreed on six samples and disagreed on six samples. Two of the disagreements involved Aroclor 1242, two involved Aroclor 1254, one involved Aroclors 1242 and 1254, and the disagreement on sample 13 involved all three Aroclors. TurboChrom suggested seven false negatives out of the 36 analytes; the DIM suggested two false negatives.

Review of the chromatogram for sample 8 shows the presence of elution peaks that correspond to the general elution times of Aroclor 1242. Review of the DIM fuzzy logic summary table for sample 8 shows that the MLRP model for Aroclor 1242 yielded an importance value that is judged to be reasonably significant. The SLE algorithm, which performs a singular value decomposition of the unknown

Table 5.12. Comparison of Aroclor identification in soil samples as determined by the DIM and TC4 software systems

\begin{tabular}{|c|c|c|c|c|c|c|c|}
\hline $\begin{array}{c}\text { Sample } \\
\text { ID }\end{array}$ & $\begin{array}{c}1242 \\
\text { by } \\
\text { DIM }\end{array}$ & $\begin{array}{c}1242 \\
\text { by } \\
\text { TC4 }\end{array}$ & $\begin{array}{c}1254 \\
\text { by } \\
\text { DIM }\end{array}$ & $\begin{array}{c}1254 \\
\text { by } \\
\text { TC4 }\end{array}$ & $\begin{array}{c}1260 \\
\text { by } \\
\text { DIM }\end{array}$ & $\begin{array}{c}1260 \\
\text { by } \\
\text { TC4 }\end{array}$ & $\begin{array}{c}\text { Agreement } \\
\text { between } \\
\text { DIM and } \\
\text { TC4 }\end{array}$ \\
\hline 1 & Yes & No & Yes & Yes & No & No & No \\
\hline 2 & No & No & No & No & Yes & Yes & Yes \\
\hline 3 & No & No & Yes & No & Yes & Yes & No \\
\hline 4 & No & No & Yes & No & Yes & Yes & No \\
\hline 5 & Yes & No & Yes & Yes & Yes & Yes & No \\
\hline 6 & No & No & Yes & Yes & Yes & Yes & Yes \\
\hline 7 & No & No & No & No & No & No & Yes \\
\hline 8 & Yes & No & No & Yes & No & No & No \\
\hline 9 & No & No & No & No & No & No & Yes \\
\hline 11 & No & No & Yes & Yes & Yes & Yes & Yes \\
\hline 12 & No & No & Yes & Yes & Yes & Yes & Yes \\
\hline 13 & Yes & No & No & Yes & Yes & No & No \\
\hline
\end{tabular}

chromatogram against the Aroclor standards, yielded an importance value that is only modestly significant. All other data analysis algorithms yielded importance values that are clearly not significant. Based on the expert knowledge of the performance of the various data processing algorithms in the DIM and the appearance of the chromatogram, it is concluded that Aroclor 1242 is present in sample 8 and that the results reported by the analyst are in error.

A review of the chromatogram for sample 13 suggests that this sample is a mixture of Aroclors 1242 and 1260. A review of the DIM fuzzy logic summary table shows that the importance value returned by the MLRP model for Aroclor 1242 was significant and that the importance values returned by the ANN and SLE algorithms are only modestly significant. Again, based on the expert knowledge related to the performance of the data processing algorithms and the appearance of the chromatogram, it is concluded 
that Aroclor 1242 is more likely to be present than Aroclor 1254, as reported by the analyst. The evidence for the presence of Aroclor 1260 in this sample is weak.

Table 5.13 summarizes the numerical values reported by the two data processing software systems. Except for sample 8, the false positives reported by the DIM are generally within a factor of two of the EQL for the PCB SAM. The same holds true for TurboChrom. Since the total Aroclor content is important from an environmental hazard perspective, the data in Table 5.13 suggest that the DIM yields results equivalent to those obtained from TurboChrom. The mean of the difference between the total Aroclor content reported by the DIM and TurboChrom is $0.06 \pm 0.26 \%$.

The limited evaluation of the DIM reported herein suggests three conclusions. First, the number of instances that the DIM and TurboChrom agreed on the analyte identy listed in Table 5.9 shows that the DIM can reduce the number of chromatograms that the analyst needs to review by $50 \%$. This would reduce the analyst labor burden. Second, the DIM may yield more false positives, or from the opposite perspective, fewer false negatives. From the perspective of environmental remediation and public health the ability to reduce the number of false positives is an important goal of the chemical analysis laboratory. Third, the numerical results show that the two software systems yield essentially equivalent results. Note that these conclusions are based on a limited set of real environmental samples and that final confirmation will require the validation of the DIM's performance with the entire set of soil samples.

Table 5.13. Individual Aroclor content in soil samples as determined by the DIM and TC4 software systems

\begin{tabular}{|c|c|c|c|c|c|c|c|c|}
\hline $\begin{array}{c}\text { Sample } \\
\text { ID }\end{array}$ & $\begin{array}{c}1242 \\
\text { by } \\
\text { DIM }\end{array}$ & $\begin{array}{c}1242 \\
\text { by } \\
\text { TC4 }\end{array}$ & $\begin{array}{c}1254 \\
\text { by } \\
\text { DIM }\end{array}$ & $\begin{array}{c}1254 \\
\text { by } \\
\text { TC4 }\end{array}$ & $\begin{array}{c}1260 \\
\text { by } \\
\text { DIM }\end{array}$ & $\begin{array}{c}1260 \\
\text { by } \\
\text { TC4 }\end{array}$ & $\begin{array}{c}\text { Total } \\
\text { by } \\
\text { DIM }\end{array}$ & $\begin{array}{c}\text { Total } \\
\text { by } \\
\text { TC4 }\end{array}$ \\
\hline 1 & 0.06 & 0 & 0.32 & 0.33 & 0 & 0 & 0.38 & 0.33 \\
\hline 2 & 0 & 0 & 0 & 0 & 0.07 & 0.09 & 0.07 & 0.09 \\
\hline 3 & 0 & 0 & 0.06 & 0 & 0.15 & 0.16 & 0.21 & 0.16 \\
\hline 4 & 0 & 0 & 0.26 & 0 & 0.46 & 0.49 & 0.72 & 0.49 \\
\hline 5 & 0.07 & 0 & 0.65 & 1.29 & 0.63 & 0.66 & 1.35 & 1.95 \\
\hline 6 & 0 & 0 & 0.38 & 0.87 & 0.49 & 0.53 & 0.87 & 1.4 \\
\hline 7 & 0 & 0 & 0 & 0 & 0 & 0 & 0 & 0 \\
\hline 8 & 0.44 & 0 & 0 & 0.19 & 0 & 0 & 0.44 & 0.19 \\
\hline 9 & 0 & 0 & 0 & 0 & 0 & 0 & 0 & 0 \\
\hline 11 & 0 & 0 & 0.10 & 0.16 & 0.15 & 0.18 & 0.25 & 0.34 \\
\hline 12 & 0 & 0 & 0.36 & 0.49 & 0.09 & 0.11 & 0.45 & 0.60 \\
\hline 13 & 0.12 & 0 & 0 & 0.10 & 0.08 & 0 & 0.20 & 0.10 \\
\hline
\end{tabular}

\subsection{SYSTEM PERFORMANCE-HARDWARE SOFTWARE ERRORS AND ALARMS}

For a consecutive period of five weeks during the operation of the PCB SAM system for the Witherspoon project, an electronic log was maintained for all error or alarm flags generated by each SLM. The purpose of this exercise was to track all problems, whether mechanical or software related, encountered during the automated operation of the PCB SAM system. The frequency and types of errors 
logged reflect the operational durability of the complete system as currently configured. Errors or alarms were generated as a result of the failure of a particular operation or SLM to complete an assigned task. This could vary from data input errors on the part of the analyst to a complete hardware failure of a specific SLM or series of SLMs.

The HCI maintains an alarm list of notices, alarms, warnings, and queries that occur while the HCI is running and attached to the TSC. To maintain records of these messages to evaluate system operational performance, the alarm list was printed on a daily basis. Hard copy archiving of the alarm list began on October 8, 1997, covering the operational periods back to October 6, 1997. The system operators were instructed to "print" this window each day to record this information for archival purposes. Limitations of the alarm list printing mechanism and various other situations occurred during the Witherspoon project that left the development team with less information than originally anticipated. The software developers discussed the possibility of using the HCI to always maintain a disk archive of the alarm list information. They decided that this functionality should be incorporated into the TSC, since the HCI is not capable of archiving any information while not connected to an operational system's TSC. Records indicating the introduction of samples into the system would also be useful information to be maintained in this log. Other reasons for the incomplete set of alarm listings include (1) the HCI computer locked up and had to be rebooted (fortunately this only happened a few days late in the project); (2) the operator inadvertently terminated the HCI program; (3) the operator failed to perform the daily "print"; and (4) the HCI printing mechanism failed to print all of the information if the list was too long.

For ease of evaluation purposes, the errors and alarms are classified into three different categories: software, operator, and hardware. Classifying the frequency and distribution of all logged errors and alarms into these categories provides a good snapshot of the system's ability, or lack thereof, to run unattended for extended periods of time under "real world" conditions. A chart of the number of errors and alarms logged by category is shown in Fig. 5.5.

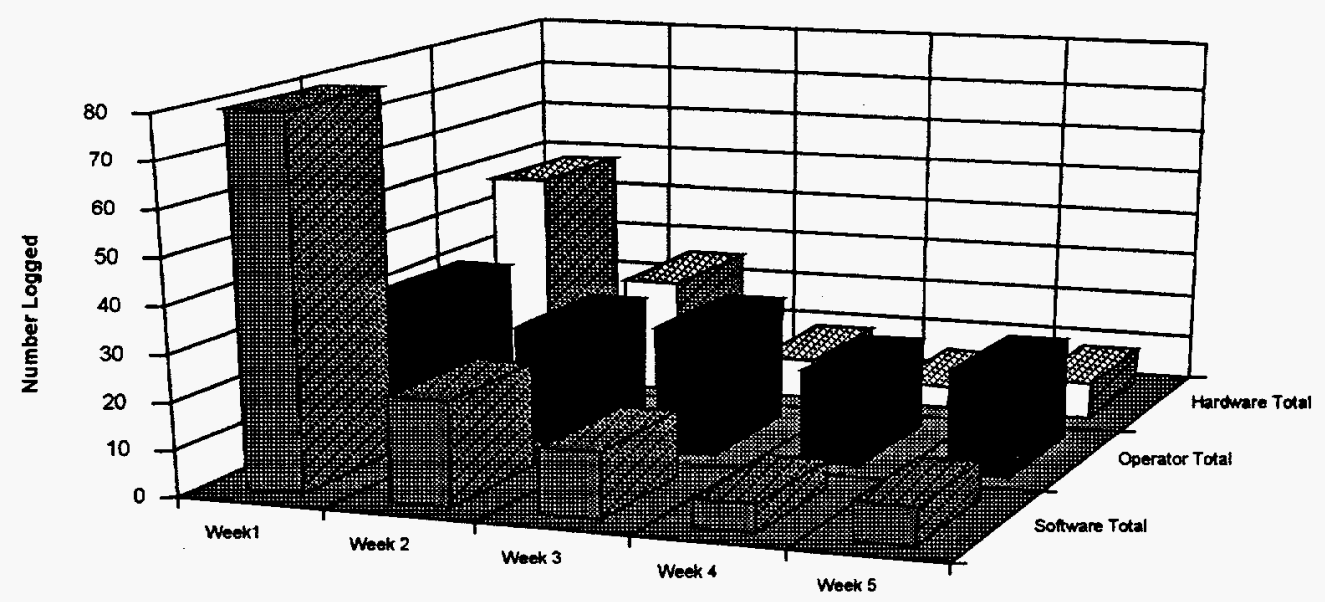

Fig. 5.5. System errors and alarms by category. 
Note that if a particular SLM encounters an error or alarm, that specific SLM is placed in a wait state until the error or alarm from the device is cleared by the analyst. This limits the possible loss of samples to the one currently being processed which reduces the number of sample reruns. All other SLM processes in the SAM continue and are not directly affected by the SLM-specific error or alarm state. Multichannel SLMs, such as the Soxhlet, shut down only the channel where the error or alarm occurred. The processing of samples continues on the remaining channels with the TSC scheduling sample processing for only those channels still operating. Thus the processing of samples continues unabated.

Errors and alarms can also be categorized as either chronic or fatal. A chronic error is one that occurs at the individual SLM level (e.g. out of solvent) and may not necessarily affect the working status of other SLMs in the SAM. Thus, the processing of samples can continue to the extent possible with the remaining operational SLMs. A fatal error is one that halts the complete operation of the SAM (e.g. a robot crash or TSC computer crash). Of all the errors and alarms encountered, only four were fatal. The robot frame reference points were lost, and the computer running the TSC crashed. A summary of the errors and alarms by SLM is shown Fig. 5.6.

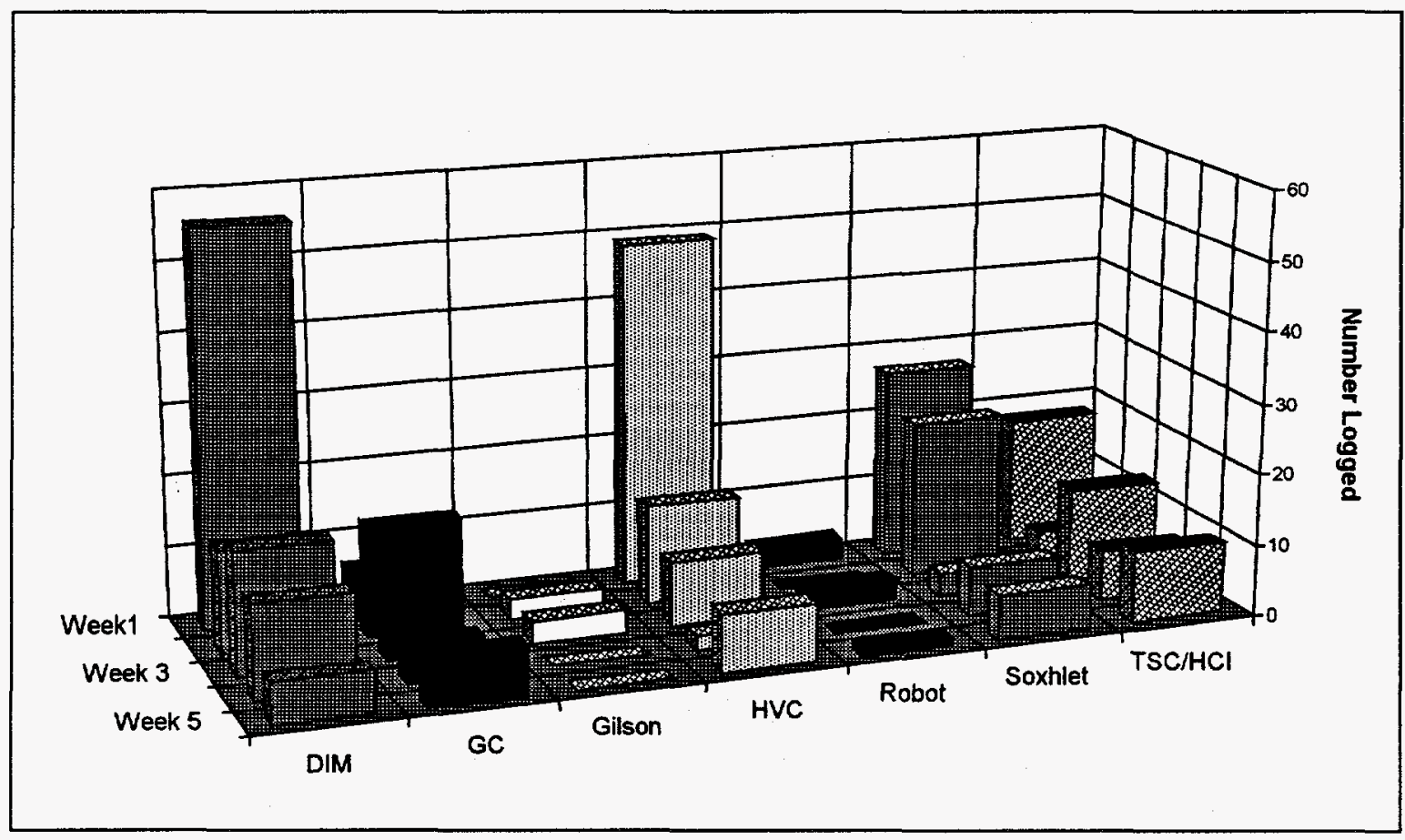

Fig. 5.6. Summary of SLM errors and alarms.

Note that the majority of alarms were recoverable. Specific problems encountered include the following:

- The only loss of samples in process occurred when the thimble was dropped while being inserted into the Soxhlet channel for processing. This occurred twice and required the repreparation of those samples: 
- A sample was aborted on the Soxhlet because of a solvent pump timeout problem on a previous sample in the same channel. This required the analyst to manually change the solvent amount, and the original value was not restored by the analyst. Subsequently, this sample was then processed manually and reintroduced into the system at the HVC. This problem led to the development team's implementing a software fix for the Soxhlet SLM.

- A sample was lost on the HVC from unknown causes. An empty vial at the completion of the HVC cycle indicated that a problem had occurred.

- On two separate occasions, one of which was recoverable, the Gilson disconnected from the TSC because of a lack of disk space.

Additional notes on alarm messages logged during the Witherspoon project are located in Appendix A.

\subsection{WORK FLOW}

The measurement of work flow and analyst time use is important to any prospective user of the CAA technology. A thorough evaluation of the labor requirements typically must wait until the betatesting phase that takes place in an operating laboratory, which will allow head-to-head comparisons of the labor required by CAA and existing approaches. Nevertheless, valuable preliminary information can be collected even at this stage of testing. The Witherspoon project gave the CAA team an opportunity to collect such data for the PCB SAM system under actual working conditions. Additional data will be collected during the beta testing of this system. However, the Witherspoon data can be used in establishing a work flow baseline for an initial cost/benefit model for the PCB SAM system.

The preparation steps of weighing, drying, and grinding are identical for both CAA and manual approaches. Time and labor measurements follow the preparation from that point on. Some steps that take little time were recorded because many small chores can add up to substantial amounts of time. A log of the analyst's time use was developed to record how the CAA analysts worked with the PCB SAM system. An observer not involved in the analysis served as a recorder of data on three separate work shifts. The time measurements, in five-minute intervals, were made on work shifts that run an entire batch of soil samples through the entire SAM. Total time is important, but how that time is broken up is almost as important. A process that requires lots of short periods of attention is much more demanding on the time of an analyst than one process that takes the same total amount of time in one block. Copies of the work sheets used to record the work flow data are included in Appendixes B and C.

The work flow data collected over three work shifts is summarized in Figs. 5.7 and 5.8 for each analyst. The work flow data was summarized into seven different task categories for ease of evaluation. The data collected for all three work shifts are plotted against the time the data points were recorded. This provides a visual representation of the actual work flow of the specific analyst as the day progressed. Analyst 1 is the CAA laboratory technician, and Analyst 2 is the CAA chemical technician, both of whom composed the CAA analytical team that processed the Witherspoon samples.

Participation in the Witherspoon project provided the CAA development team with an opportunity to collect labor and work flow data while "real world" soil samples were being processed by the analytical team. These data can be used to assess whether using a fully automated PCB SAM system is economically competitive with current commercial laboratory practices. In theory, labor accounts for a large portion of the cost of performing an analysis. Therefore, any significant reduction in labor afforded by an automated system would directly impact the price of the analysis, or the profit margin, as the case may be. 
The work flow data collected during the operation of the PCB SAM system was used to estimate the CAA PCB SAM price per sample for PCB analysis in soil. The analysis was performed on the basis of labor and material costs only. The CAA Witherspoon analytical team was limited to a single work shift operational schedule because of institutional safety and health policies. The PCB SAM system could not be left unattended overnight, and the buddy system was required after normal facility working hours. Until the CAA development team collects more comparative data from commercial laboratory operations, this is meant to be a "back of the envelope" type of calculation. Using this as a baseline for the CAA PCB SAM system, further price reductions per sample scenarios are generated using realistic performance projections given a specific set of resources and system performance enhancements. These projections are represented in Fig. 5.9.

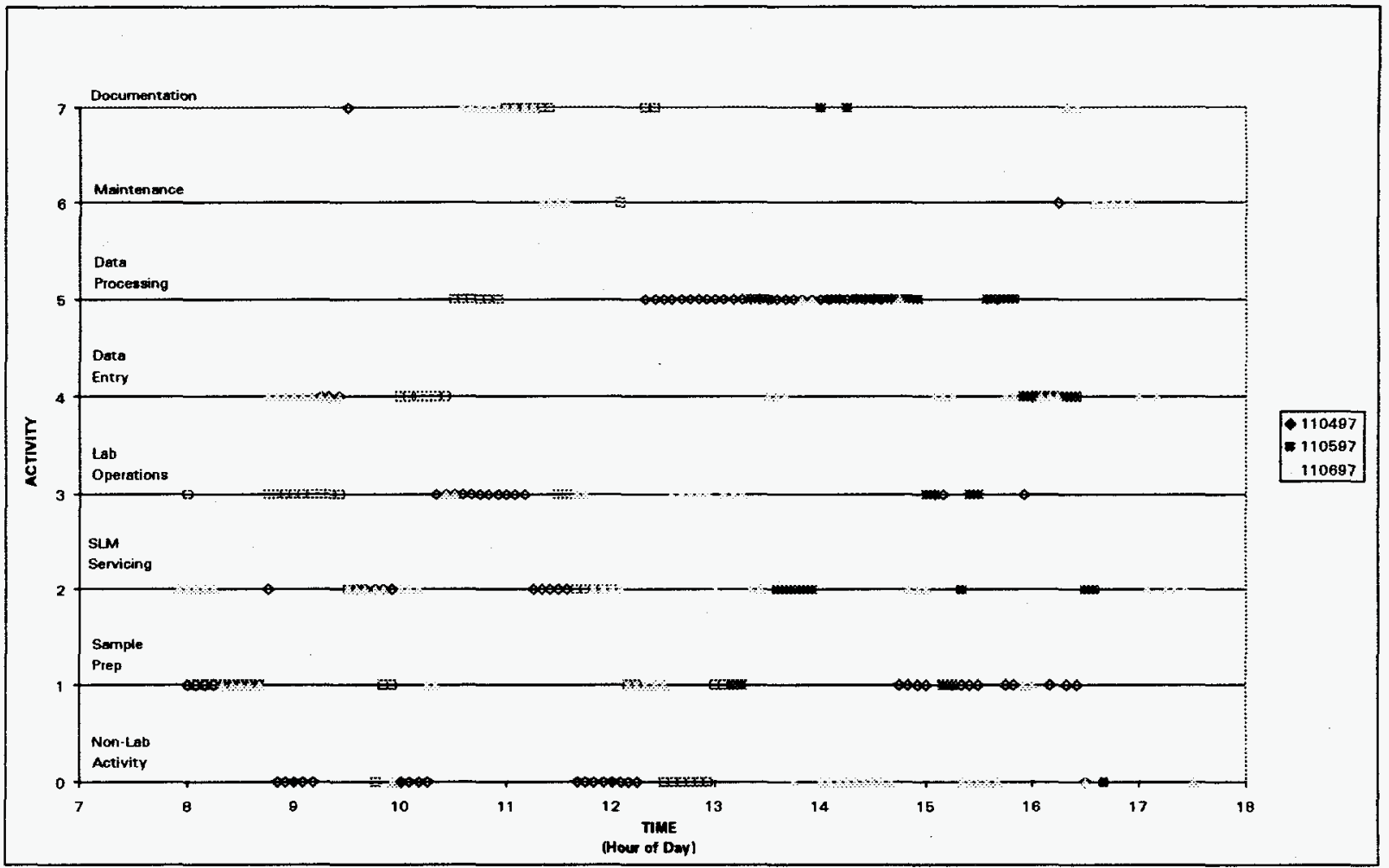

Fig. 5.7. Graphical representation of work flow for analyst 1.

Price-reduction scenarios include the following:

1. Incorporating system improvements and modifications based on the Witherspoon experience (System Mods). This includes electronic management of data and the automated generation of data packages.

2. Operating the system in a true automated environment where unattended operation becomes the norm (Functioning Lab Use). This would eliminate the buddy system employed by the CAA team, as required by institutional health and safety regulations. 
3. Fostering the use of multiple shifts for the feeding and caring of the system (Run 2 Shifts Unattended).

4. Configuring functional system enhancements (Add DilutionReRun Feature).

Based on these projections and conditions, a fully functional CAA PCB SAM system is projected to bring the price of a PCB analysis in soil to below the $\$ 50 /$ sample price range. The commercial laboratory persample contract price for the Witherspoon project was $\$ 82$.

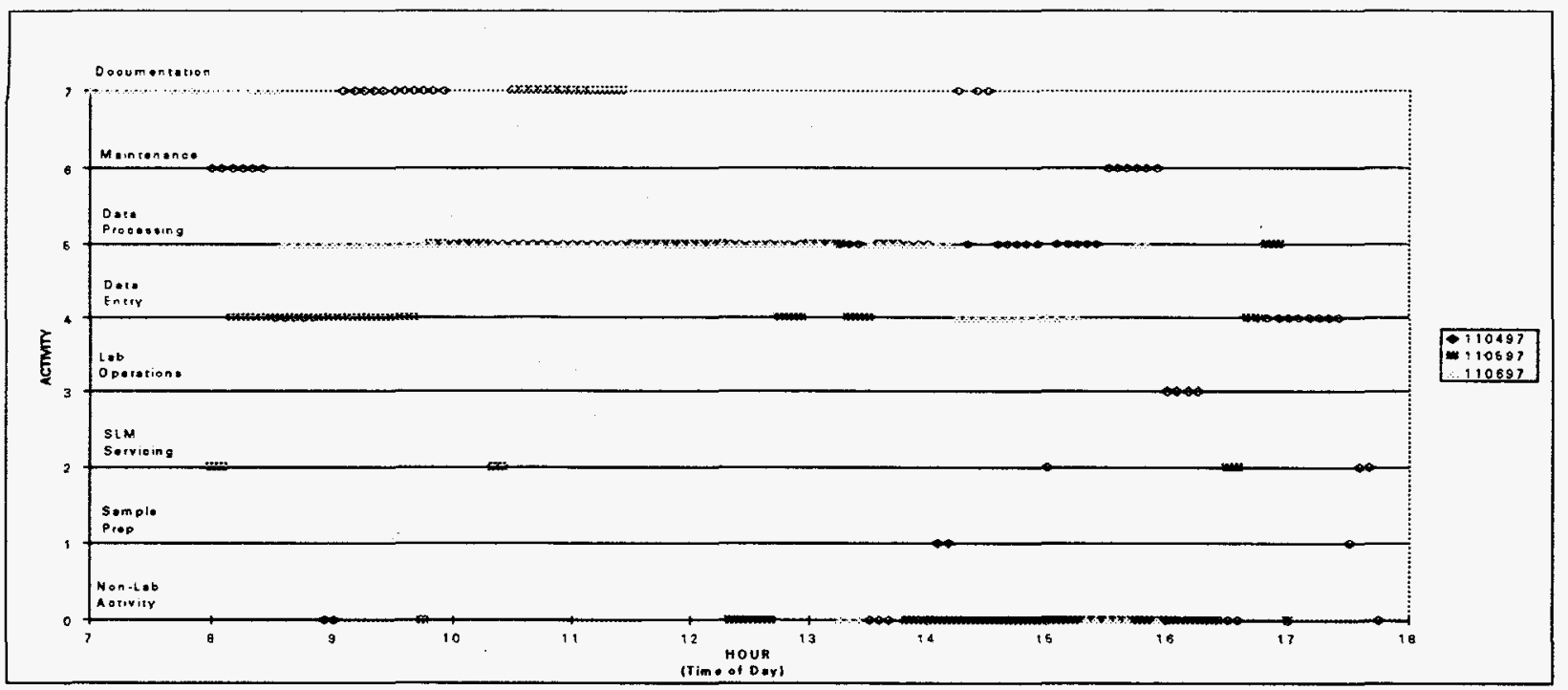

Fig. 5.8. Graphical representation of work flow for analyst 2

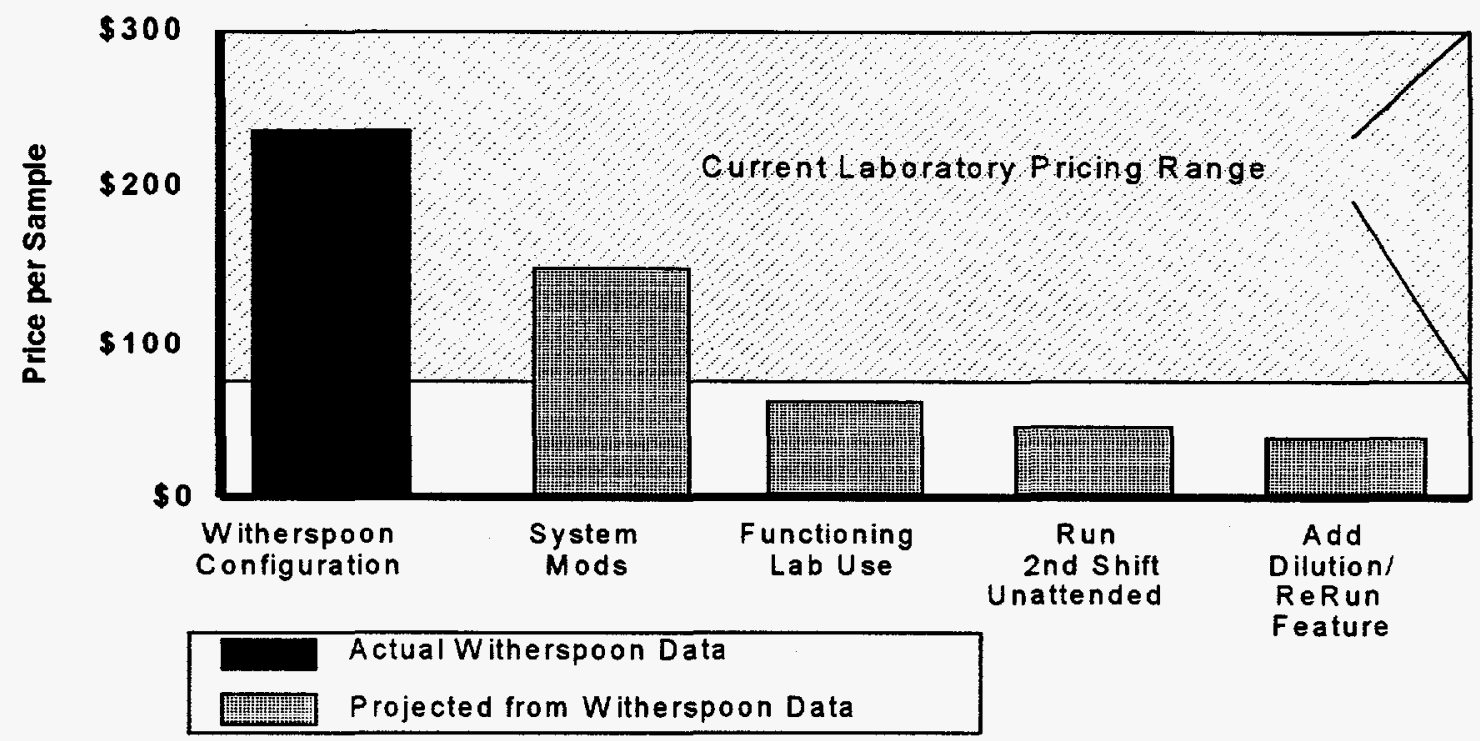

Fig. 5.9. Preliminary cost projections for CAA PCB system. 


\section{AREAS IDENTIFIED AS NEEDING FURTHER DEVELOPMENT}

By assessing the type and number of errors and alarms logged by the system, the development team can determine the operational readiness of the system for the next phase of testing and or technology demonstration deployment. The Witherspoon technology demonstration was an excellent experience for the development team as several areas were identified where additional development work is needed. Errors logged early in the operation of the system were caused by operator inexperience with the system and SLM sensor problems that required additional adjustments. As a general rule, the individual hardware SLMs performed exceptionally well once the sensor adjustments were made. After solving problems encountered at the startup of the Witherspoon project, the hardware required minimal intervention on the part of the analysts.

The biggest area of concern was the user interface and data management software, or lack thereof. The development team quickly realized that the user interface software was designed more for use by the developers than for ease of use by the analysts. The majority of software errors were attributable to the fact that the data input requirements were not obvious to the analysts using the system in a fully automated mode. Data format requirements, redundancy of data input required by multiple software screens, convoluted error recovery requirements, and data manipulation practices all contributed to problems encountered by the analysts in using the PCB SAM system in an automated mode.

It was interesting to observe how the production-oriented nature of the analysts pushed the performance envelope of the PCB SAM system. The time limiting step in the PCB method is the two-hour extraction performed at the beginning of the method. This creates a two-hour wait for the rest of the SLMs in the PCB SAM system before processing can begin downstream, even though the SLMs are capable of performing work. Rather than being satisfied with the completion of a batch at the end of the work shift, the analysts devised a way to start the next day's extractions during their current shift and continue the sample processing with the remaining SLMs the next morning. The system was originally designed with a series of queuing racks for each SLM, with each rack capable of queuing up to 15 samples per rack per SLM operation. The process of adding more samples to a batch than the system was capable of queuing at any one time created some interesting TSC scheduling problems. As this capability was not originally included in the software, the analysts were forced to develop a manual process to manipulate vessels within the queuing racks during the initial startup of the system each day. Needless to say, it took them a while to get used to this work-around, and errors resulted when they forgot to perform a critical task, such as replacing a beaker in the queue rack. It is debatable whether the additional throughput of two to four samples per batch was worth the labor requirements created by the analysts as part of the work-around to the PCB SAM system shortcomings. Clearly, the development team thought that 15 queuing slots were adequate. The real world experience proved otherwise.

The real-world testing of the CAA PCB SAM system through participation in the Witherspoon project provided the CAA development team with several valuable lessons learned:

1. User Interface-The "Keep It Simple Stupid" axiom is directly applicable to the design of a user interface that is intuitive to analytical laboratory personnel. The initial operation of the PCB SAM system was hindered by the analyst's need to learn the operational idiosyncrasies of the software. Although a substantial amount of capabilities exist in the software, the user interface required a higher level of working knowledge on the part of the analyst compared with graphical user interfaces typically encountered by the analysts when using commercial software packages. The development team's perception of the ease of use did not match the real-world experience of the analysts operating the PCB SAM system. 
2. Data Manager Functionality-Although a data manager is scheduled for development and inclusion into the CAA system paradigm, one did not exist at the time of the Witherspoon test. The need for a data manager SLM was strongly reinforced during this project. The data manager will eliminate multiple data entry and data file naming convention problems encountered by the analysts during the course of this project. This will also include better management of data flow to and from those modules that need the information. The analyst should have to enter pertinent data only once. The system should manage the flow of that data to the resources requiring the information. Development of a data manager has become a high priority for the development team as a result of this project.

3. Simple Decision-Making Capability-Because of the high percentage of soil samples that contained mid to high levels of PCBs, a significant amount of time was spent either diluting extracts into the range of the calibration curve or reextracting a smaller aliquot of the original sample. Although the system software has the ability to flag outlier data (e.g., exceeding QCs limits and the calibration range of the measurement system), the system does not have the ability to make simple dilution decisions, perform the dilution, and reinsert the diluted extract into the processing stream for analysis. The development team did not have an appreciation for the usefulness of such a capability until the PCB SAM system was operated under a realworld environment.

4. Hardware-Focused Development-The hardware performed exceptionally well under the Witherspoon test conditions. This can be credited to the development team's effort in designing, building, testing, and integrating the hardware SLMs into a functional SAM system. It is evident from this experience that the software, although very capable and functional, was not developed at the same pace or to the same functional level as the hardware. Clearly, additional resources must be spent in software development before the PCB SAM system can be commercialized. 


\section{CONCLUSIONS}

The CAA project is developing standardized, software-driven, site-deployable robotic laboratory systems with the objective of lowering the per-sample analysis cost, decreasing sample turnaround time, and minimizing human exposure to hazardous and radioactive materials associated with DOE remediation projects. The first integrated system developed by the CAA project is designed to determine PCB content in soil matrices. A demonstration and development testing of this system was conducted in conjuction with surface soil characterization activities at the David Witherspoon 1630 Site in Knoxville, Tennessee.

The CAA system used in the Witherspoon project consisted of five hardware SLMs, one software SLM, the TSC, and the HCI. Four of the hardware SLMs included a four-channel Soxhlet extractor, an HVC, a column cleanup, and a GC. These SLMs performed the sample preparation and measurement steps within the total analysis protocol. The fifth hardware module was a robot that transports samples between the SLMs and the required consumable supplies to the SLMs. The software SLM is an automated DIM that receives raw data from the GC SLM and analyzes the data to yield the analyte information. The TSC is a software system that provides the scheduling, management of system resources, and the coordination of all SLM activities. The HCI is a graphical user interface that presents the automated laboratory to the analyst in terms of the analytical procedures and methods. Human control of the automated laboratory is accomplished via the HCI. Sample information required for processing by the automated laboratory is entered through the HCI. Information related to the sample and the system status is presented to the analyst via graphical icons.

The demonstration and development testing of the automated PCB laboratory was conducted according to and evaluated against the benchmark methods defined in the EPA "Test Methods for Evaluating Solid Waste, Physical/Chemical Methods, SW-846" manual. The specific methods implemented are Methods 3500, "Organic Extraction and Sample Preparation," Method 3541, "Soxhlet Extration," Method 3600, "Clean-up," Method 3620, "Florisil Column Clean-up," Method 8000, "Gas Chromatography," and Method 8082, "Polychlorinated Biphenyls by Capillary Gas Chromatography."

A total of 234 field split samples were received from September 19, 1997, to November 13, 1997. The samples were also sent to a commercial laboratory; the results reported by the commercial laboratory will be used in the remedial investigation report of the David Witherspoon 1630 Site. A total of 340 extracts were processed through the system, including 26 method blanks, 29 blind QC samples, and 24 matrix spike/spike duplicates. The samples were processed during 24 working days, averaging 9.8 extractions per day. A total of 807 extracts were processed through the GC and DIM SLMs, which included 19 calibration standards, 111 daily calibration check standards, and 337 dilutions and reruns of the original extracts. PCBs were detected in more than $60 \%$ of the soil samples; this is uncharacteristically high for a typical remediation effort.

The chemical performance of the automated PCB laboratory was evaluated according to the QA and QC measures specified in SW-846, Chapter One, Sect. 4.4, "Laboratory QA and QC Procedures," as well as specific criteria listed in the methods noted previously. Performance parameters included:

- blind QC standards,

- method blanks,

- daily calibration check standards, 
- matrix spike and matrix spike duplicates,

- $\quad$ surrogate recovery,

- $\quad$ field duplicate samples,

- $\quad$ MDLs, and

- results data reported by the commercial laboratory.

The CAA system met or exceeded all the laboratory QA and QC requirements. Average recovery for the blind QC samples was $100 \pm 32 \%$. Method blanks revealed that contamination in the system was above the regulatory acceptance criterion on one occasion, and that occurred after a sample containing a total Aroclor content of $64 \mathrm{ppm}$ was processed. Out of the 111 daily calibration standard checks, only one failed the 80 to $120 \%$ acceptance criterion. The mean recovery for the matrix spike and matrix spike duplicates was $110 \pm 34 \%$. The mean surrogate recovery across all sample types (control, blank, standard, and unknown) was $74 \pm 16 \%$. The mean difference for six field duplicates that contained a measurable Aroclor content was $34 \pm 27 \%$ for the CAA system and $48 \pm 36 \%$ for the commercial laboratory. A paired $t$-test applied to the soil sample results obtained from the CAA system and the commercial laboratory showed that the two data sets were not statistically different. The MDL was $0.02 \mathrm{ppm}$, which exceeds the proficiency guideline of Method 8082 by about a factor of 3 .

Early in the project, the contractor performing the remedial investigation recognized the quality of the CAA data and used the CAA data as a quality check on the contract laboratory. Normally, this quality check task is performed by sending about 10 to $15 \%$ of the samples, as field splits, to a second laboratory.

Although the DIM SLM ran during the entire Witherspoon project, its results were used only as a guide by the operators because the DIM had not been validated. A post-Witherspoon project review effort shows that the DIM did not yield any false negatives on the blind QC samples, while the TurboChrom software system yielded three false negatives. The DIM may be yielding more false positives, particularly at Aroclor concentrations near the EQL. The DIM may also yield fewer false negative results, which is an important attribute from the environmental remediation and public health perspectives. Based on a limited subset of soil samples reviewed after the completion of the Witherspoon project, the DIM may save approximately $50 \%$ of the chemist's effort that is devoted to reviewing raw chromatograms.

The frequency of system hardware and software errors and alarms showed a marked decrease as the project proceeded. A large number of DIM-related errors that occurred during the first week of the project were associated with a problem in the DIM control software. After this software problem was corrected, essentially all the DIM-related alarms and errors were traceable to operator errors (i.e., entry of incorrect information associated with the sample when it was entered into the $\mathrm{HCI}$ or failure to add the retention time markers to diluted extracts). Most of the other errors were associated with the operator's unfamiliarity with the HCI. Only four samples $(\sim 0.3 \%$ of the total number of extractions) were lost because of system errors while the samples were being processed; in these cases, the manual sample preparation steps were repeated and the sample was reintroduced into the system. 
Work flow and task time data were collected during the project. These data were used to make a preliminary per-sample cost projection for the CAA PCB SAM; a cost of $\$ 50$ per-sample should be achievable. The commercial laboratory per-sample contract price for the Witherspoon project was $\$ 82$.

The Witherspoon project identified areas needing further development. The major need is the development of a totally electronic capability to manage the data and to generate the data packages. The second need involves modifying the HCI so that it more closely meets the intuitive needs of the chemists. The third need involves the addition of simple decision capability to the TSC. This enhancement would allow the system to automatically perform dilutions and resubmissions of the diluted extract to the GC SLM.

The hardware systems performed exceptionally well. The chemists reported that they had never processed so many samples in such a short time frame with so few equipment problems. 
APPENDIX A 


\section{APPENDIX A. SUMMARY OF THE TYPES OF ALARMS AND ERRORS ENCOUNTERED DURING THE WITHERSPOON PROJECT}

1. XXX Command Overdue-This is a message indicating that the task sequence controller (TSC) has not heard from the standard laboratory module (SLM) in the period that the SLM told the TSC that it would provide a communication. For the data interpretation module (DIM), Soxhlet and high-volume concentrator (HVC) SLMs, this is a software-induced problem. Normally the command is eventually completed, and no operator intervention is required. The software developer fixed this problem in the DIM after week one. For the HVC and the Soxhlet, it appeared to be an artifact of the out-of-solvent alarm being signaled. For the robot this is a major problem because the vendor-supplied robot control software cannot signal the client code that it has completed the transport of an item. This problem occurred about every two days. Consequently, the robot's PC was rebooted every morning to avoid this problem. In other words, after every $\mathrm{X}$ number of transactions, the robot control software hangs.

2. XXX Expired intra laboratory unit operation (ILUO)-This message indicates that a command is overdue and a status command from the TSC to find out why the command is overdue was not returned by the SLM. The internals of the SLM have quit working, but the connection is still open. This is an SLM software problem.

3. DIM Error in Set-This indicates a sample data entry error on the part of the analyst.

4. DIM Generic Error-This message from the DIM notifies the analyst that the surrogate recovery quality assurance test failed. For all practical purposes this is not a system or SLM error; rather, it is a message that suggests a problem with the chemistry being executed by the standard analysis method (SAM). A significant number of these were logged when the surrogate was not added to dilutions and when the HVC became clogged and was outputting only the makeup solvent.

5. XXX Sample Aborted-The analyst aborted the sample for one reason or another. Approximately half of these were inadvertent errors caused by the analyst's not knowing how to recover from the situation.

6. Gas Chromatograph (GC) Destination Data File Already Exists-Analyst has duplicated a previously used sample ID number. This is a sample entry error.

7. GC Source Data File Does Not Exist-This is a software data transfer error. The GC client software uses only the first seven characters of the Varian Star data filename instead of the full eight characters allowed under the Windows 3.1 operating system.

8. GC Network Common Data Format (netCDF) Comment Insertion Failed-This is a software problem in the $\mathrm{GC}$ client, and the specific cause is unknown.

9. GC Errors-Toward the end of the project, the GC computer disk became full resulting in some of these errors. Also, when a sample was aborted, the GC was not properly unloaded. The software was unaware of the sample being removed from the $\mathrm{GC}$ and assumed the sample was where the software had last left it. The operator did not know how to recover from this error state. 
10. Gilson Port Is Occupied-Whenever the TSC crashed, the Gilson Column Cleanup SLM reconnected to the TSC with a sample already positioned in its sample entry port. The analyst did not know how to properly recover from this situation.

11. HVC 10-mL Vial Found-After a robot crash, the system was restarted with a vial left in the HVC port. This is a result of operator inexperience.

12. XXX Out of Solvent-This problem was resolved after week two. This error was a result of improperly refilling the solvent kegs. The software threshold setting for sensing an out-of-solvent condition was secondary to the initial cause (reporting out of solvent when there was adequate solvent present). Some of these errors were also the result of refilling a solvent keg and not priming the Soxhlet or HVC solvent pumps.

13. HVC Pickup Movement Time Out-This is a hardware error that requires some resolution on the part of the development team. It started to occurr intermittently at week five.

14. Network Command Overdue-This is a rare and inconsistent condition that never manifested itself before the Witherspoon project. Many of the SLMs were reporting overdue alarms. This is possibly caused by a network traffic problem. The cause is undetermined. A flurry of these errors occurred twice during the Witherspoon project.

15. Robot Module Execution Error-This usually indicates that the robot has collided into something and that required action could not be referenced to the proper location.

16. A lot of the sample aborts were caused by errors in sample information data entry. This is an operator error.

17. Soxhlet Solvent Pump Timeout-This is a hardware/software problem that the development team has been unable to track down. A work-around was supplied to make it very easy to continue the extraction process.

18. Soxhlet Inert Gas Pressure Out of Range-The gas supply valve to the Soxhlet SLM was inadvertently turned off. This situation occurred only once, but the message was sent multiple times.

19. TSC Crash-There were actually seven TSC crashes: two caused by extra commas in the spreadsheet comma separated file used to input a batch of samples into the human-computer interface (HCI), three caused by the HCI computer hanging up from an unknown Windows 95 operating system or application error, and two because of remote HCI communication problems caused by a new network security implementation at the Idaho National Engineering and Environmental Laboratory.

20. TSC Errors-These resulted from operator interaction and sample data entry problems. 
APPENDIX B 


\section{APPENDIX B: WORK SHEET USED TO ACQUIRE TASK TIME DATA}

Recorded By:

Date:

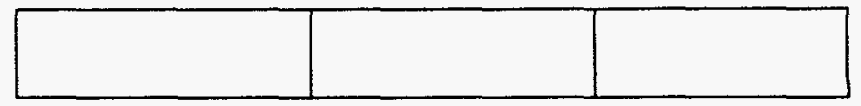

\begin{tabular}{|c|c|c|c|}
\hline !" & 1 & 2 & 3 \\
\hline \multicolumn{4}{|l|}{ Batch size } \\
\hline \multicolumn{4}{|l|}{ Number of samples } \\
\hline \multicolumn{4}{|l|}{$\begin{array}{l}\text { Number of blanks or other quality control } \\
\text { samples }\end{array}$} \\
\hline \multicolumn{4}{|l|}{ Start up } \\
\hline \multicolumn{4}{|l|}{ Check Solvents } \\
\hline \multicolumn{4}{|l|}{ (add solvents if required) } \\
\hline \multicolumn{4}{|l|}{ Check gases } \\
\hline \multicolumn{4}{|l|}{ (replace gas cylinders if required) } \\
\hline \multicolumn{4}{|l|}{$\begin{array}{l}\text { Start each SLM and check for proper } \\
\text { operation }\end{array}$} \\
\hline \multicolumn{4}{|l|}{ Soxhlet } \\
\hline \multicolumn{4}{|l|}{ Concentrator } \\
\hline \multicolumn{4}{|l|}{ Column } \\
\hline \multicolumn{4}{|l|}{ GC } \\
\hline \multicolumn{4}{|l|}{ DIM } \\
\hline \multicolumn{4}{|l|}{ Robot } \\
\hline \multicolumn{4}{|l|}{ Sample preparation } \\
\hline \multicolumn{4}{|l|}{ Add samples to thimbles } \\
\hline \multicolumn{4}{|l|}{ Add all spikes to samples } \\
\hline \multicolumn{4}{|l|}{ SLM } \\
\hline \multicolumn{4}{|l|}{ Add thimbles to racks } \\
\hline \multicolumn{4}{|l|}{ Add Soxhlet beakers to racks } \\
\hline \multicolumn{4}{|l|}{ Add concentrator beakers to racks } \\
\hline \multicolumn{4}{|l|}{ Add concentrator vials to racks } \\
\hline \multicolumn{4}{|l|}{ Add column vials to racks } \\
\hline \multicolumn{4}{|l|}{ Add column cartridges to rack } \\
\hline \multicolumn{4}{|l|}{ End of shift clean up of all of the above } \\
\hline \multicolumn{4}{|l|}{ Data Entry } \\
\hline \multicolumn{4}{|l|}{ Initialize TSC computer } \\
\hline \multicolumn{4}{|l|}{ Enter sample data, ID, method, priority } \\
\hline \multicolumn{4}{|l|}{ Out of Ordinary } \\
\hline $\begin{array}{l}\text { Recovery from any abnormal conditions } \\
\text { (describe and note the time) }\end{array}$ & & & \\
\hline
\end{tabular}


APPENDIX C 
APPENDIX C: WORK SHEET USED TO ACQUIRE WORK-FLOW DATA

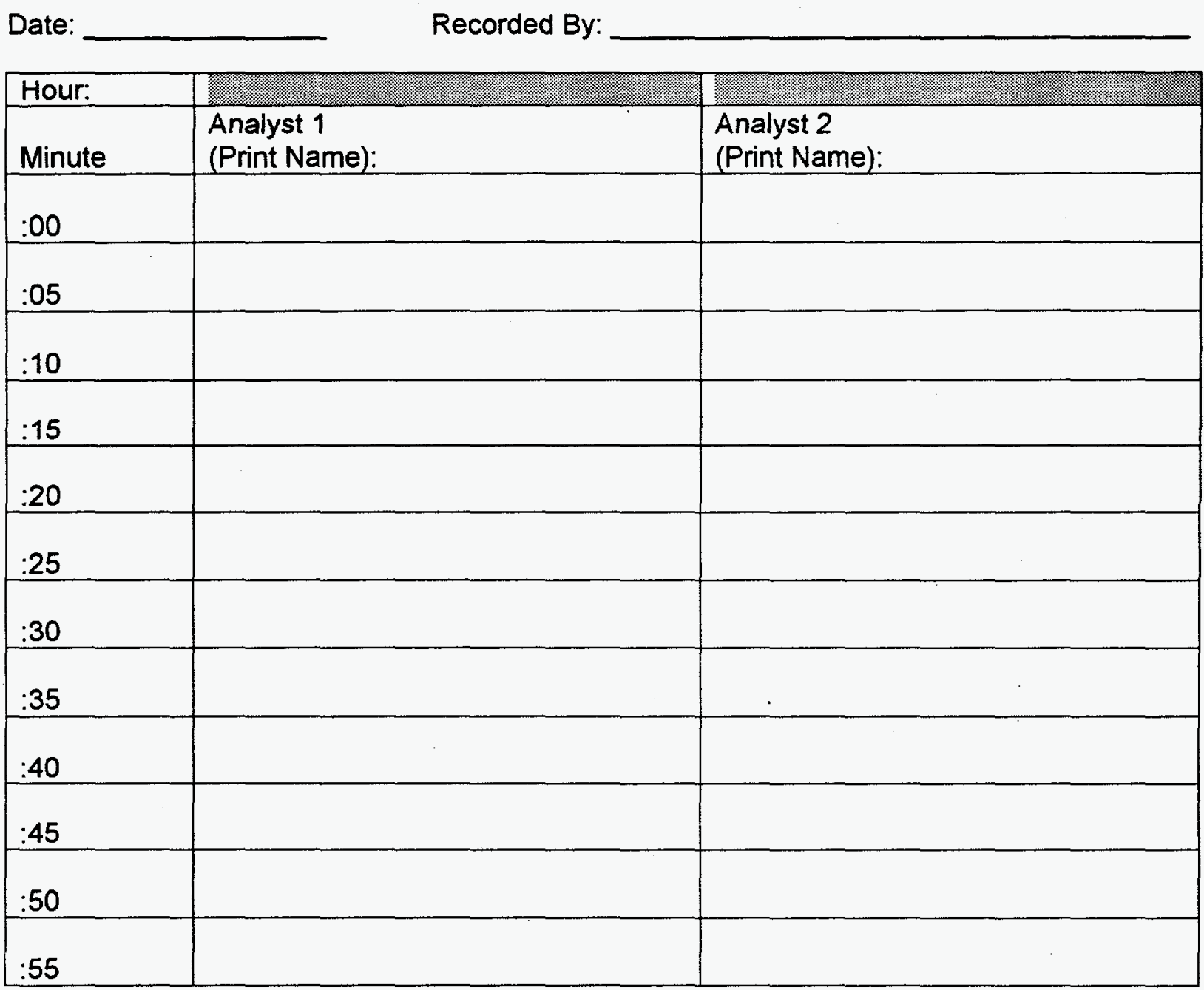


ORNL/TM-13590

\title{
INTERNAL DISTRIBUTION
}

\author{
1. J. F. Birdwell \\ 2. J. G. Dorsey \\ 3. J. A. Ealy \\ 4. D. C. Haley \\ 5. J. N. Herndon \\ 6. M. A. Hunt \\ 7-11. L. N. Klatt \\ 12. A. P. Malinauskas \\ 13. T. E. Myrick \\ 14. K. E. Plummer \\ 15. J. D. Randolph \\ 16. S. L. Schrock \\ 17. S. P. Singh \\ 18. P. L. Szczygiel \\ 19. B. B. Spencer \\ 20. D. H. Thompson \\ 21. D. J. Trent \\ 22. H. R. Yook \\ 23. J. R. Younkin \\ 24. D. S. Zingg \\ 25. Central Research Library \\ 26. ORNL Laboratory Records-RC \\ 27-28. ORNL Laboratory Records-OSTI

\section{EXTERNAL DISTRIBUTION} \\ 29. M. Abidi, Department of Electrical and Computing Engineering, \\ University of Tennessee, Knoxville, TN 37996-2100 \\ 30. P. Adsit, Department of Mechanical Engineering, \\ University of Florida, Gainesville, FL 32611-6300 \\ 31. G. Boyd, US Department of Energy \\ Office of Science and Technology EM-50 \\ 1000 Independence Avenue SW, Washington, DC 20585 \\ 32. D. M. Carden, DOE Oak Ridge Operations Office \\ P.O. Box 2001, Oak Ridge, TN 37831 \\ 33. M. Clark, Idaho National Engineering \& Environmental Laboratory, \\ P.O. Box 1625, MS 2220,Idaho Falls, ID 83415-2220 \\ 34. C. Crane, Department of Mechanical Engineering, \\ University of Florida, Gainesville, FL 32611-6300 \\ 35. D. E. Dale, Los Alamos National Laboratory \\ P.O. Box 1663, MS K484, Los Alamos, NM 87545 \\ 36. J. DeGregory, US Department of Energy \\ 19901 Germantown Road, Germantown, MD 20874-1290 \\ 37. T. Goode, Jacobs Engineering, Oak Ridge, TN 37830
}


38. K. Hakonson, Los Alamos National Laboratory

P.O. Box 1663, MS K484, Los Alamos, NM 87545

39. R. Hirsch, APTI, $125024^{\text {th }}$ Street, Washington, DC 20037

40. M. Houck, Jacobs Engineering, Oak Ridge, TN 37830

41-50. K. Humphrey, Los Alamos National Laboratory P.O. Box 1663, MS J580, Los Alamos, NM 87545

51. R. Kinoshita, Idaho National Engineering \& Environmental Laboratory P.O. Box 1625, MS2220, Idaho Falls, ID 83415-2220

52. R. M. Hollen, Los Alamos National Laboratory P.O. Box 1663, MS J580, Los Alamos, NM 87545

53. M. Monagle, Advanced Industrial Chemistry Corp. 9220 Jill Patricia NW, Albuquerque, NM 87114

54. J. Moore, DOE Oak Ridge Operations Office P.O. Box 2001, Oak Ridge, TN 37831

55. J. Noble-Dial, DOE Oak Ridge Operations Office P.O. Box 2001, Oak Ridge, TN 37831

56. Ivan C. Oelrich 1801 N. Beauregard Street, Alexandria, VA 22311

57. A. Perkins, DOE Oak Ridge Operations Office P.O. Box 2001, Oak Rige, TN 37831

58. J. Riveria, Sandia National Laboratory P.O. Box 5800, Department 9622, MS 0660, Albuquerque, NM 87185-1004

59. R. Rubin, Institute for Defense Analysis, 1801 N. Beauregard Street, Alexandria, VA 22311

60. C. Rzeszutko, Los Alamos National Laboratory P.O. Box 1663, MS J580, Los Alamos, NM 87545

61. F. Settle, 1960 West Drive, Lexington, VA 24450

62. D. Shumate, Robins Science \& Environmental Laboratory WR-ALC/TIEL, Robins, AFB, GA 31098-1637

63. R. Shurtliff, Idaho National Engineering \& Environmental Laboratory P.O. Box 1625, Idaho Falls, ID 83415-2220

64. J. Tulenko, Department of Mechanical Engineering, University of Florida, Gainesville, FL 32611-6300

65. T. Turner, Idaho National Engineering \& Environmental Laboratory P.O. Box 1625, MS 2220, Idaho Falls, ID 83415-2220

66. D. Wadsworth, Idaho National Engineering \& Environmental Laboratory P.O. Box 1625, MS 2220, Idaho Falls, ID 83415-2220

67. J. S. Walker, DOE-HQ, US Department of Energy 19901 Germantown Road, Germantown, MD 20874

68. L. Yarbrough, DOE Albuquerque Operations Office P.O. Box 5400, Albuquerque, NM 87185-5400

69. J. Young, Idaho National Engineering \& Environmental Laboratory P.O. Box 1625, MS 2220, Idaho Falls, ID 83415-2220 